H. Tapia-Recillas

Nagoya Math. J.

Vol. 69 (1978), 65-96

\title{
ULTRAMETRIC THETA FUNCTIONS AND ABELIAN VARIETIES
}

\author{
HORACIO TAPIA-RECILLAS ${ }^{(*)}$
}

Let $k$ be a field complete with respect to a non-trivial, non-archimedean valuation and let $g$ be a positive integer. Consider the following question: if $\Gamma$ is a multiplicative subgroup of $G_{g}=\left(k^{*}\right)^{g}$ satisfying certain "Riemann conditions", can one construct in a natural way an abelian variety defined over $k$ having $G_{g} / \Gamma$ as its set of $k$-rational points? This problem was first considered by Morikawa [3]. J. Tate provided a complete solution for $g=1$ (cf. for example [6]). J. McCabe [2] gave a partial solution for $g>1$. He showed how to attach to $\Gamma$ a graded ring $R$ of theta functions such that $A=\operatorname{Proj.} R$ is a $g$-dimensional abelian variety over $k$. He further constructed a homomorphism $\varphi: G_{g} / \Gamma \rightarrow A_{k}$ and showed that it is injective. But he could only prove that $\varphi$ is surjective under restrictive hypotheses, assuming that $k$ is locally compact of characteristic zero. Recently Raynaud [5], Gerritzen [1] and Mumford [4] have generalized and completely solved the problem we are considering. But their techniques are non-elementary and it is still perhaps interesting to show that the map $\varphi$ is surjective within the context of Tate-McCabe theory, using only simple calculations with Laurent power series.

That is the goal of this paper.

Let ord.: $k^{*} \rightarrow$ Reals denote the order function associated to our valuation. In part 1 we start with a $g \times g$ matrix $\left(\mathscr{A}_{i j}\right)$ with entries in $k^{*}$ satisfying the following Riemann conditions: $\mathscr{A}_{i j}=\mathscr{A}_{j i}$ and the associated matrix (ord. $\mathscr{A}_{i j}$ ) is positive definite. Following McCabe we construct the ring $R$ of theta functions associated to $\left(\mathscr{A}_{i j}\right)$, the abelian variety $A$ and the map $\varphi: G_{g} / \Gamma \rightarrow A_{k}$ where $\Gamma$ is the multiplicative sub-

Received July 2, 1976.

(*) Thi $i$ paper includes parts of the author's Ph. D. dissertation at Brandeis University. I would like to thank Professor Paul Monsky for his suggestions during the writing of the thesis and the preparation of this paper. This work was supported by CONACYT PNCB 000070 and the Centro de Investigación del I.P.N., México. 
group of $G_{g}$ generated by the column vectors of $\left(\mathscr{A}_{i j}\right)$.

Part II is the heart of the paper. In it we assume that the offdiagonal elements of $\left(\mathscr{A}_{i j}\right)$ have order 0 . We call this case the "diagonal case". Here the reduction $\bar{A}$ of $A$ plays an important role. For $g=1$ $\bar{A}$ is a rational curve with an ordinary double point; in general $\bar{A}$ is a rational variety which looks very much like a product of such curves. We attach to each $P \in A_{k}$ a certain subset $S(P)$ of $\{1,2, \cdots, g\} ; S(P)$ describes how singular $\bar{P}$ is on $\bar{A}$. We say that $P$ is a unit point if $S(P)$ $=\varnothing$; this means that $\bar{P}$ is non-singular on $\bar{A}$. In $\S$ II. 3 we use an implicit function type argument to show that $\varphi$ is 1-1 and that all unit points are in the image of $\varphi$. The proof that any $P \in A_{k}$ is in $\varphi\left(G_{g}\right)$ is by induction on the cardinality of $S(P)$. The key steps in the induction are an addition formula on $A$, (Theorem II. 4.6), and the "decomposition theorem", (Theorem II. 6.6), whose proof depends on the study of the zeroes of a certain Laurent series $\theta_{P}$.

We return to the general case in part III. Using the diagonal case and an isogeny argument we show that $\varphi$ is bijective, assuming only that each ord. $\mathscr{A}_{i j}$ is rational. This mild restriction is unnecessary as Gerritzen's result show, but we have been unable to avoid it.

Throughout this paper we use the following notation: $k$ is a field complete with respect to a non-trivial, non-archimedean valuation, ord : $k^{*} \rightarrow$ Reals is the associated order function, $\mathcal{O}, \mathscr{M}$ and $\bar{k}$ are the valuation ring, maximal ideal and residue class field of the valuation. $U$ is the unit group of $\mathcal{O}$ and $G_{g}$ is the product of $g$ copies of $k^{*}$.

\section{I}

Part $I$ is concerned with the definition and basic properties of the ring of theta functions $R$. It contains a proof that $A=\operatorname{Proj} . R$ is an abelian variety of dimension $g$ over $k$.

Most of this material can be found in the first three chapters of McCabe [2], but our arguments are somewhat simpler.

\section{§.1. The ring of theta-functions}

A Laurent series over $k$ is a formal sum $\sum_{I \in Z^{g}} \mathscr{A}_{I} X^{I}, \mathscr{A}_{I} \in k$, which converges for all $\left(x_{1}, \cdots, x_{g}\right) \in G_{g}$. (we shall use standard multivariable notation throughout. If $I=\left(i_{1}, \cdots, i_{g}\right)$ then $X^{I}$ means $\left.\prod_{j} X_{j}^{i_{j}}\right)$. The Laurent series form a $k$-algebra $\mathscr{L}$. The subring of $\mathscr{L}$ consisting of 
series with $\mathscr{A}_{I} \in \mathcal{O}$ is called $\mathscr{L}_{0} . \mathscr{L}$ is a domain, and if an element of $\mathscr{L}$ vanishes on all of $G_{g}$, each $\mathscr{A}_{I}=0$. Suppose $n>1$ and $(r)=\left(r_{1}, \cdots, r_{g}\right)$ $\in(Z / n Z)^{g}$. An element $\sum \mathscr{A}_{I} X^{I}$ of $\mathscr{L}$ is said to have $n$-parity $(r)$ if $\mathscr{A}_{I}=0$ unless each $i_{j}$ reduces to $r_{j} \bmod . n$. Let $\mathscr{L}^{(r)}$ denote the subspace of elements of $\mathscr{L}$ having $n$-parity $(r)$. Then we get a decomposition $\mathscr{L}=\oplus_{(r)} \mathscr{L}^{(r)}$; the " $n$-parity decomposition of $\mathscr{L}^{\text {". }}$

Let $\left(\mathscr{A}_{i j}\right)$ be a $g \times g$ symmetric matrix with entries in $k^{*}$ such that the associated matrix (ord. $\mathscr{A}_{i j}$ ) is positive definite.

Let $V_{j}=\left(\mathscr{A}_{j 1}, \cdots, \mathscr{A}_{j g}\right), q_{j}=\mathscr{A}_{j j}$. If $m>0, R_{m}\left(\mathscr{A}_{i j}\right)$ (or just $R_{m}$ ) will denote the set of elements $\theta \in \mathscr{L}$ which satisfy the following functional relation:

$$
\theta\left(V_{j} X\right)=q_{j}^{-m} X_{j}^{-2 m} \theta(X) \quad j=1,2, \cdots, g .
$$

Note that if $\theta(X)=\sum b_{I} X^{I} \in R_{m}$ and $V_{j}^{I}=\prod_{t=1}^{g} \mathscr{A}_{j t}^{i_{t}}$ it follows from the relation $(P)$ that the $b_{I}$ 's satisfy:

$$
b_{I} V_{j}^{I} q_{j}^{m}=b_{I+2 m \delta_{j}} \quad j=1,2, \cdots, g
$$

where $\delta_{j}=(0, \cdots, \underset{(j)}{0,1,0, \cdots, 0)}$

THEOREM I.1.1. Let $m>0$ and $\mathscr{L}=\oplus_{(r)} \mathscr{L}^{(r)}$ be the 2m-parity decomposition of $\mathscr{L}$. If $R_{m}^{(r)}=R_{m} \cap \mathscr{L}^{(r)}$, then:

1) $R_{m}^{(r)}$ is a 1-dimensional k-vector space.

2) $R_{m}=\oplus_{(r)} R_{m}^{(r)}$ and $\operatorname{dim}_{k} R_{m}=(2 m)^{g}$

3) $R=\oplus_{0}^{\infty} R_{m}$ is a graded k-algebra with $R_{0}=k$.

Proof. Suppose $\sum b_{I} X^{I} \in R_{m}^{(r)}$. Using the relation $\left(P^{\prime}\right)$ we see that $b_{I}$ determines $b_{I^{\prime}}$ for $I \equiv I^{\prime} \bmod .2 m$. Thus $\operatorname{dim} . R_{m}^{(r)} \leq 1$. To complete the proof of 1 ) we exhibit a generator of $R_{m}^{(r)}$. Take representatives of $r_{j}$ in $Z$ and by abuse of language call them $r_{j}$ too. If $i_{j}=2 m t_{j}+r_{j}$, set

$$
b_{I}=\prod_{j=1}^{g} q_{j}^{t_{j}\left(m t_{j}+r_{j}\right)} \prod_{j>\ell} \mathscr{A}_{j \ell}^{i_{j} t_{\ell}+r_{\ell} t_{j}}
$$

and let $b_{I}=0$ if $I \not \equiv(r) \bmod .2 m$. Set $\varphi(X)=\sum b_{I} X^{I}$.

A calculation shows that the $b_{I}$ satisfy $\left(P^{\prime}\right)$. Also

$$
\text { ord. } \begin{aligned}
b_{I} & =\sum_{j} t_{j}\left(m t_{j}+r_{j}\right) \text { ord. } q_{j}+\sum_{j>\ell}\left(r_{j} t_{\ell}+r_{\ell} t_{j}+2 m t_{j} t_{\ell}\right) \text { ord. } \mathscr{A}_{j \ell} \\
& =m \sum_{j, \ell} t_{j} t_{\ell} \text { ord. } \mathscr{A}_{j \ell}+\sum_{j, \ell} r_{j} t_{\ell} \text { ord. } \mathscr{A}_{j \ell}
\end{aligned}
$$


Since the matrix (ord. $\mathscr{A}_{i j}$ ) is positive definite, $\varphi \in \mathscr{L}$ and 1) is proved. 2) and 3) are obvious.

The decomposition of $R_{m}$ in the Theorem is called the $2 m$-parity decomposition of $R_{m}$ and $R$ is called the graded ring of Theta functions associated to the matrix $\left(\mathscr{A}_{i j}\right)$.

There is a relation between the graded rings $R\left(\mathscr{A}_{i j}\right)$ and $R\left(\mathscr{A}_{i j}^{n}\right)$, $n>0$, that we shall make constant use of. Namely:

$$
\begin{aligned}
& \theta \in R_{m}\left(\mathscr{A}_{i j}\right) \Rightarrow \theta \in R_{m n}\left(\mathscr{A}_{i j}^{n}\right) \\
& \theta \in R_{m}\left(\mathscr{A}_{i j}^{n}\right) \Rightarrow \theta\left(X^{n}\right) \in R_{m n}\left(\mathscr{A}_{i j}\right) .
\end{aligned}
$$

These are easily verified. Using (a) together with Theorem I. 1.1. we get:

Proposition I.1.2. If $n$ is a fixed positive integer, $S_{m}=R_{m n}\left(\mathscr{A}_{i j}^{n}\right)$ is a k-vector space of dimension $(2 \mathrm{mn})^{g}, S=\oplus_{0}^{\infty} S_{m}$ is a graded k-algebra, $R_{m} \subseteq S_{m}$ and $R$ is a subring of $S$.

Next using (b) with $m$ replaced by $m n$ we see that $\theta(X) \rightarrow \theta\left(X^{n}\right)$ defines a graded homomorphism $S \rightarrow R$ of degree $n^{2}$. The restriction of this map to $R$ is a graded endomorphism of $R$ of degree $n^{2}$. Both of these maps will be denoted by $\alpha_{n}$. A dimension count shows that $\alpha_{n}(S)$ consists precisely of those elements of $R$ that can be written as Laurent series in $X_{j}^{n}, j=1,2, \cdots, g$.

THEOREM I.1.3. $R$ is integral over $\alpha_{n}(R)$.

Proof. We first show that $S$ is integral over $R$. Let $\theta \in S_{m}$. For $1 \leq i \leq g$ let $T_{i}(\theta)=q_{i}^{m} X_{i}^{2 m} \theta\left(V_{i} X\right)$. Then :

$$
\begin{aligned}
T_{i}(\theta)\left(V_{i}^{n} X\right) & =q_{i}^{m}\left(q_{i}^{n} X_{i}\right)^{2 m} \theta\left(V_{i}^{n}\left(V_{i} X\right)\right) \\
& \left.=\left(q_{i}^{m} X_{i}^{2 m} q_{i}^{2 m n}\right)\left(q_{i}^{-m n^{2}}\left(q_{i} X_{i}\right)^{-2 m n}\right) \theta\left(V_{i} X\right)=q_{i}^{-m n^{2}} X_{i}^{-2 m n}\right) T_{i}(\theta) .
\end{aligned}
$$

Thus $T_{i}(\theta) \in S_{m}$ for $i=1,2, \cdots, g$ and we have defined operators $T_{i}: S_{m} \rightarrow S_{m}$. An easy induction shows that $T_{i}^{\ell}(\theta)(X)=q_{i}^{m \ell} X_{i}^{2 m \ell} \theta\left(V_{i}^{\ell} X\right)$, for all $\ell$. Thus $T_{i}^{n}$ is the identity map on $S_{m}$. Also

$$
\left(T_{i} \circ T_{j}\right)(\theta)=T_{i}\left(q_{j}^{m} X_{j}^{2 m} \theta\left(V_{j} X\right)\right)=q_{i}^{m} X_{i}^{2 m} q_{j}^{m} \mathscr{A}_{i j}^{2 m} X_{j}^{2 m} \theta\left(V_{j} V_{i} X\right) .
$$

Since this is symmetric in $i$ and $j$, the $T_{i}$ commute.

For each $i$, the various $T_{i}: S_{m} \rightarrow S_{m}$ fit together to give a graded 
automorphism of $S$ which we also denote by $T_{i}$. Let $T$ be the finite group generated by the automorphisms $T_{i}$. By the definition of $T_{i}, R$ is the subring of invariants of $S$ under $T$. So, $S$ is integral over $R$ and $\alpha_{n}(S)$ over $\alpha_{n}(R)$. It remains to show that every $\theta \in R$ is integral over $\alpha_{n}(S)$. We may assume that $\theta$ is in some $R_{m n}$ and has a definite $n$ parity. But then $\theta^{n}$ is a Laurent series in the $X_{i}^{n}$, lies in $\alpha_{n}(S)$, and the theorem is proved.

Now let $E$ be the $g \times g$ matrix all of whose entries are 1 . Then the $2 g \times 2 g$ matrix $\left(\begin{array}{cc}\mathscr{A}_{i j} & E \\ E & \mathscr{A}_{i j}\end{array}\right)$ clearly satisfies the Riemann conditions. Let $R^{\prime}$ be the graded ring of theta-functions attached to this matrix. We shall label the Laurent series variables by $X_{1}, \cdots, X_{g}, Y_{1}, \cdots, Y_{g}$ instead of $X_{1}, \cdots, X_{2 g}$. Then a Laurent series $\theta(X, Y)$ is in $R_{m}^{\prime}$ if and only if :

$$
\begin{aligned}
& \theta\left(V_{j} X, Y\right)=q_{j}^{-m} X_{j}^{-2 m} \theta(X, Y) \\
& \theta\left(X, V_{j} Y\right)=q_{j}^{-m} Y_{j}^{-2 m} \theta(X, Y) .
\end{aligned}
$$

In particular, if $\theta$ and $\varphi$ are elements of $R_{m}$, then $\theta(X) \varphi(Y)$ is in $R_{m}^{\prime}$ and we get a map $R_{m} \otimes_{k} R_{m} \rightarrow R_{m}^{\prime}$.

Proposition I.1.4. The above map is bijective; thus $R^{\prime}$ is the 2-fold Segré product of $R$ with itself over $k$.

Proof. Injectivity is clear. To prove ontoness it suffices to construct elements of pre-assigned $2 m$-parity in the image of $R_{m} \otimes R_{m}$. This may be done by taking $\theta(X) \varphi(Y)$ where $\theta$ and $\varphi$ have the desired $2 m$ parities.

The following proposition is the key to the construction of a group law on $A=$ Proj. $(R)$.

Proposition I.1.5. If $\theta \in R_{m}^{\prime}$ then $\theta^{\prime}(X, Y)=\theta\left(X Y, X Y^{-1}\right) \in R_{2 m}^{\prime} . \quad \theta \rightarrow \theta^{\prime}$ defines a graded endomorphism $\beta$ of $R^{\prime}$ of degree 2 . $\beta \circ \beta$ maps $\theta$ to $\theta\left(X^{2}, Y^{2}\right)$ and $R^{\prime}$ is integral over $\beta\left(R^{\prime}\right)$.

Remark. $\theta\left(X Y, X Y^{-1}\right)$ is shorthand for

$$
\theta\left(X_{1} Y_{1}, \cdots, X_{g} Y_{g}, X_{1} Y_{1}^{-1}, \cdots, X_{g} Y_{g}^{-1}\right) .
$$

Proof.

$$
\theta^{\prime}\left(V_{j} X, Y\right)=\theta\left(V_{j} X Y, V_{j} X Y^{-1}\right)=q_{j}^{-m}\left(X_{j} Y_{j}\right)^{-2 m} q_{j}^{-m}\left(X_{j} Y_{j}^{-1}\right)^{-2 m} \theta
$$


Since $\left(X_{j} Y_{j}\right)\left(X_{j} Y_{j}^{-1}\right)=X_{j}^{2}$ we get the first functional equation for $\theta^{\prime}$. Similarly, using the fact that $(X Y)\left(X^{-1} Y\right)=Y^{2}$ we get the second, and $\theta^{\prime} \in R_{2 m}^{\prime}$. We see at once that $\beta$ is a degree 2 endomorphism and that $\beta \circ \beta=\alpha_{2}$. By Theorem I.1.3, with $R$ replaced by $R^{\prime}, R^{\prime}$ is integral over $\beta\left(R^{\prime}\right)$.

For technical reasons connected with characteristic 2 we shall also need a 4-fold Segré product. The $4 g \times 4 g$ matrix which has 4 copies of $\left(\mathscr{A}_{i j}\right)$ down its diagonal and all 1's elsewhere satisfies the Riemann conditions. Let $R^{\prime \prime}$ be the corresponding graded ring of theta-functions. Label the Laurent series variables by $X_{1}, \cdots, X_{g}, Y_{1}, \cdots, Y_{g}, Z_{1}, \cdots, Z_{g}$, $T_{1}, \cdots, T_{g}$. The proof of Proposition I.1.4, gives:

Proposition I.1.6. The natural map $R_{m} \otimes R_{m} \otimes R_{m} \otimes R_{m} \rightarrow R_{m}^{\prime \prime}$ is bijective and $R^{\prime \prime}$ is the 4-fold Segré product of $R$ with itself over $k$.

Proposition I.1.7. If $\theta \in R_{m}^{\prime \prime}$ then

$$
\theta^{\prime \prime}(X, Y, Z, T)=\theta\left(X Y Z, X Z^{-1} T, X Y^{-1} T^{-1}, Y Z^{-1} T^{-1}\right) \in R_{3 m}^{\prime \prime} .
$$

$\theta \rightarrow \theta^{\prime \prime}$ defines a degree 3 graded endomorphism $\eta$ of $R^{\prime \prime} . \quad \eta \circ \eta=\alpha_{3}$ and $R^{\prime \prime}$ is integral over $\eta\left(R^{\prime \prime}\right)$.

Proof. Similar to that of Proposition I.1.5 and based on the identities:

$$
\begin{aligned}
& (X Y Z)\left(X Z^{-1} T\right)\left(X Y^{-1} T^{-1}\right)=X^{3} \\
& (X Y Z)\left(X^{-1} Y T\right)\left(Y Z^{-1} T^{-1}\right)=Y^{3} \\
& (X Y Z)\left(X^{-1} Z T^{-1}\right)\left(Y^{-1} Z T\right)=Z^{3} \\
& \left(X Z^{-1} T\right)\left(X^{-1} Y T\right)\left(Y^{-1} Z T\right)=T^{3} .
\end{aligned}
$$

Remark. The proof of Proposition I.1.5 essentially rests on the fact that $A \circ A^{t}=2 I$ where $A$ is the matrix $\left(\begin{array}{rr}1 & 1 \\ 1 & -1\end{array}\right)$. Similarly, Proposition I.1.7 uses the fact that $B \circ B^{t}=3 I$ where

$$
B=\left(\begin{array}{rrrr}
1 & 1 & 1 & 0 \\
1 & 0 & -1 & 1 \\
1 & -1 & 0 & -1 \\
0 & 1 & -1 & -1
\end{array}\right) .
$$

\section{§ I.2. Finite generation of $R$}

In this section we show that the graded ring of theta functions is 
a finitely generated algebra over $k$. In the course of the proof stronger results are obtained, namely:

(1) if char. $k \neq 2, R_{2}$ generates $R_{2 m}$ for large $m$.

(2) if char. $k \neq 3, R_{3}$ generates $R_{3 m}$ for large $m$.

LEMMA I.2.1. The elements of $R_{1}$ have no common zero in $G_{g}$.

Proof. Let $q_{j}=\mathscr{A}_{j j}$; by extending $k$ we may assume $q_{j}=b_{j}^{2}$ with $b_{j} \in k^{*}$. If $I=\left(i_{1}, \cdots, i_{g}\right), i_{j}=2 m t_{j}+r_{j}$, let

$$
C_{I}=\prod_{j=1}^{g} b_{j}^{i_{j}^{2}} \prod_{r>s} \mathscr{A}_{r s}^{i_{r} i_{s}}
$$

and set $\varphi(X)=\sum C_{I} X^{I}$.

Since ord. $C_{I}=\frac{1}{2} \sum_{j} i_{j}^{2}$ ord. $q_{j}+\sum_{r>s} i_{r} i_{s}$ ord. $\mathscr{A}_{r s}$ and the matrix (ord. $\mathscr{A}_{i j}$ ) is positive definite, $\varphi \in \mathscr{L}$. Clearly we have:

$$
C_{I+\dot{o}_{j}}=C_{I} \cdot b_{j}^{2 i_{j+1}} \prod_{s \neq j} \mathscr{A}_{s j}^{i_{s j}} .
$$

Thus

$$
\left(\prod_{s=1}^{g} \mathscr{A}_{j s}^{i_{s}}\right) C_{I}=b_{j}^{-1} C_{I+\delta_{j}}
$$

and

$$
\varphi\left(V_{j} X\right)=b_{j}^{-1} X_{j}^{-1} \varphi(X) \quad j=1,2, \cdots, g .
$$

Let $\theta(X, Y)=\varphi(X Y) \varphi\left(X Y^{-1}\right)$. The proof of Proposition I.1.5 shows that $\theta \in R_{1}^{\prime}$. So $\theta$ is in the image of $R_{1} \otimes R_{1}$. Now suppose all the elements of $R_{1}$ vanish at some point $x \in G_{g}$. Then $\theta(x, Y)=0$, so $\varphi(x Y)$ $\cdot \varphi\left(x Y^{-1}\right)=0$. But $\mathscr{L}$ is an integral domain and $\varphi \neq 0$, so the lemma follows.

THEOREM 1.2.2. Let $m>0$. Then the elements of $R_{m}$ which are power series in $X_{i}^{m}$ have no common zero in $G_{g}$.

Proof. Let $x$ be any element of $G_{g}$. By Lemma I.2.1 there is a $\theta \in R_{1}\left(\mathscr{A}_{i j}^{m}\right)$ such that $\theta\left(x^{m}\right) \neq 0$. Then by the remark preceding Proposition I.1.2. $\theta\left(X^{m}\right) \in R_{m}\left(\mathscr{A}_{i j}\right)$ and does not vanish at $x$.

Let $n>0$. We assume for now that char. $k$ does not divide $n$ and the group $U_{n}$ of $n$-th roots of unity is contained in $k$. Recall that $S_{m}$ $=R_{m n}\left(\mathscr{A}_{i j}^{n}\right)$. 
For $m>0$ and $u=\left(u_{1}, \cdots, u_{g}\right) \in U_{n}^{g}$ let $R_{m, u}$ denote the set of elements $\theta \in \mathscr{L}$ satisfying the following functional relation:

$$
\theta\left(V_{j} X\right)=u_{j} q_{j}^{-m} X_{j}^{-2 m} \theta(X) \quad j=1,2, \cdots, g
$$

Proposition I.2.3. Let $n, U_{n}$ and $S_{m}$ be as above. Then each $R_{m, u}$ is a subspace of $S_{m}$ of dimension $(2 m)^{g}$ and $S_{m}=\oplus_{(u)} R_{m, u},(u) \in U_{n}^{g}$.

Proof. Let $T_{i}: \mathscr{L} \rightarrow \mathscr{L}$ be the operators of Theorem I.1.3. If $\theta \in R_{m, u}$ then $T_{i}(\theta)=u_{i} \theta$ and $T_{i}^{n}(\theta)=\theta$. By the proof of Theorem I.1.3, $S_{m}$ is the subspace of $\mathscr{L}$ fixed by the $T_{i}^{n}$, so $R_{m, u} \subset S_{m}$. Also the $R_{m, u}$ are just the subspaces of $S_{m}$ corresponding to the various irreducible representations of the group $T$. So $S_{m}=\oplus_{(u)} R_{m, u}$. The proof that $\operatorname{dim} R_{m, u}$ $=(2 m)^{g}$ is similar to that of Theorem I.1.1. We omit it.

Proposition I.2.4. With the same notation as above, the elements of $R_{m, u}$ of pre-assigned n-parity have no common zero in $G_{g}$.

Proof. Let $(r)=\left(r_{1}, \cdots, r_{g}\right)$ be a given $n$-parity. Suppose $\theta \in R_{n}$ with trivial $n$-parity. By extending $k$ we can get $C_{j} \in k^{*}$ such that

$$
C_{j}^{2 n}=\left(\prod_{t=1}^{g} \mathscr{A}_{j t}^{r_{t}}\right) \cdot u_{j}^{-1} \quad j=1,2, \cdots, g .
$$

If $(C)=\left(C_{1}, \cdots, C_{g}\right)$, set $\varphi(X)=\left(\prod_{j=1}^{g} X_{j}^{r_{j}}\right) \cdot \theta(C X)$. Then $\varphi\left(V_{j} X\right)$ $=\left(\prod_{t=1}^{g} \mathscr{A}_{j t}^{r_{t}} X_{t}^{r_{t}}\right) q_{j}^{-n}\left(C_{j} X_{j}\right)^{-2 n} \theta(C X)$ and it follows that $\varphi \in R_{n, u}$ with $n$ parity $(r)$.

The zeroes of $\varphi$ are just translates of the zeroes of $\theta$ by $C^{-1}$. But, by Theorem I.2.2, the $\theta \in R_{m}$ with trivial $n$-parity have no common zero.

COROLlaRY 1.2.5. If $m$ is a multiple of $n$, the elements of $R_{m, u}$ of pre-assigned n-parity have no common zero in $G_{g}$.

Proof. If $(r)$ is the given $n$-parity and $x \in G_{g}$, choose $\theta_{1} \in R_{n}$ with trivial $n$-parity such that $\theta_{1}(x) \neq 0$ and $\theta_{2} \in R_{n, u}$ with $n$-parity $(r)$ such that $\theta_{2}(x) \neq 0$. If $m=n p, \theta_{1}^{p-1} \theta_{2} \in R_{m, u}$ and has $n$-parity $(r)$.

The following simple lemma will be used to prove the finite generation of $R$.

LEMMA I.2.6. Let $M$ be a graded algebra over a field $k$. Assume: $M_{m}=0$ for all negative $m, M_{m}$ is finite dimensional over $k$ for all $m$ and there is a polynomial $P$ such that $\operatorname{dim} M_{m}=P(m)$ for all large $m$. Then, if $M_{1}$ generates $M_{m}$ for infinitely many $m$, it generates $M_{m}$ for all large $m$. 
Proof. Let $\tilde{M}$ be the subalgebra of $M$ generated by $M_{1}$ and $\tilde{P}$ be the Hilbert polynomial of $\tilde{M}_{m}$. By assumption, $M_{m}=\tilde{M}_{m}$ for infinitely many $m$. Thus $P$ and $\tilde{P}$ are equal at infinitely many $m, P=\tilde{P}$ and $\operatorname{dim} M_{m}=\operatorname{dim} \tilde{M}_{m}$ for large $m$.

Suppose now that we are in the situation of Proposition I.2.3 with $n=2$. In other words, we assume that char. $k \neq 2$.

Proposition I.2.7. If char. $k \neq 2$ and $n=2$, then $S_{1}$ generates $S_{m}$ for all large $m$.

Proof. It suffices to show that each $\theta \in S_{2 t}$ is in $S_{t} \cdot S_{t}$. For then $S_{1}$ generates $S_{2 r}$ for all $r$ and we can use Lemma I.2.6.

By Proposition I.2.3 we may assume $\theta \in R_{2 t, u}$ for some $u \in U_{2}^{g}$, and that $\theta$ has a definite 2-parity. Choose $\theta_{1} \in R_{2 t, u}$ with the same 2-parity as $\theta$ so that $\theta_{1}(1) \neq 0$. (see Cor. I.2.5). Let $\varphi(X, Y)=\theta(X Y) \theta_{1}\left(X Y^{-1}\right)$. It is easy to see that $\varphi \in R_{4 t}^{\prime}$. Since $\theta$ and $\theta_{1}$ have the same 2-parity, $\varphi$ is a power series in $X_{i}^{2}, Y_{i}^{2}$ and therefore is in $\alpha_{2}\left(S_{t}\right) \otimes \alpha_{2}\left(S_{t}\right)$, (cf. remark after Proposition I.1.2). Thus,

$$
\varphi(X, X)=\theta_{1}(1) \theta\left(X^{2}\right)=\theta_{1}(1) \alpha_{2}(\theta) \in \alpha_{2}\left(S_{t}\right) \cdot \alpha_{2}\left(S_{t}\right) ; \quad \theta \in S_{t} \cdot S_{t}
$$

and we are done.

THEOREM I.2.8. If char. $k \neq 2, R_{2}\left(\mathscr{A}_{i j}\right)$ generates $R_{2 m}\left(\mathscr{A}_{i j}\right)$ for all large $m$, and the graded subring $R_{(2)}=\oplus_{0}^{\infty} R_{2 m}$ of the ring of theta functions is a finitely generated k-algebra.

Proof. By extending $k$ we may assume $\mathscr{A}_{i j}=b_{i j}^{2}$ with $b_{i j} \in k^{*}$ and $b_{i j}=b_{j i}$. Since $S_{m}\left(b_{i j}\right)=R_{2 m}\left(\mathscr{A}_{i j}\right)$, the first part comes from Prop. I.2.7, and the second part follows.

THEOREM I.2.9. If char. $k \neq 2$, the ring $R$ of theta functions is a finitely generated $k$-algebra.

Proof. Since multiplication by a non-zero element of $R_{1}$ gives an isomorphism of the $R_{(2)}$-module $\oplus_{0}^{\infty} R_{2 m+1}$ with an ideal in $R_{(2)}, \oplus_{0}^{\infty} R_{2 m+1}$ is a finite $R_{(2)}$-module. So $R$ is a finite $R_{(2)}$-module and a finitely generated $k$-algebra.

We now treat the case of characteristic 2. More generally we suppose that char. $k \neq 3$. We take $n=3$ and assume temporarily that $U_{3} \subset k$. 
Proposition I.2.10. With the assumptions above, $S_{1}$ generates $S_{m}$ for all large $m$.

Proof. As in the proof of Prop. I.2.7, it suffices to show that each $\theta \in S_{3 t}$ is in $S_{t} \cdot S_{t} \cdot S_{t}$. We may assume that $\theta \in R_{3 t, u}$ and has a definite 3-parity. Choose $\theta_{1} \in R_{3 t, u}$ with the same 3-parity as $\theta$ so that $\theta_{1}(1) \neq 0$. Choose $\theta_{2} \in R_{3 t}$ with trivial 3-parity so that $\theta_{2}(1) \neq 0$. Set

$$
\varphi(X, Y, Z, T)=\theta(X Y Z) \theta_{1}\left(X Z^{-1} T\right) \theta_{1}\left(X Y^{-1} T^{-1}\right) \theta_{2}\left(Y Z^{-1} T^{-1}\right) .
$$

It is easily seen that $\varphi \in R_{9 t}^{\prime \prime}$ and is a power series in $X_{i}^{3}, Y_{i}^{3}, Z_{i}^{3}, T_{i}^{3}$, so it lies in the image of $\alpha_{3}\left(S_{t}\right) \otimes \alpha_{3}\left(S_{t}\right) \otimes \alpha_{3}\left(S_{t}\right) \otimes \alpha_{3}\left(S_{t}\right)$. Then $\varphi(X, X, X, 1)$ $=\theta_{1}^{2}(1) \theta_{2}(1) \theta\left(X^{3}\right)$ is in $\alpha_{3}\left(S_{t}\right) \cdot \alpha_{3}\left(S_{t}\right) \cdot \alpha_{3}\left(S_{t}\right)$ and so $\theta \in S_{t} \cdot S_{t} \cdot S_{t}$.

THEOREM I.2.11. If char. $k \neq 3$ then $R_{3}\left(\mathscr{A}_{i j}\right)$ generates $R_{3 m}\left(\mathscr{A}_{i j}\right)$ for all large $m$, and $\oplus_{0}^{\infty} R_{3 m}$ is a finitely generated k-algebra.

Proof. By extending $k$ we may assume that $U_{3} \subset k$ and that $\mathscr{A}_{i j}$ $=b_{i j}^{3}$ with $b_{i j} \in k^{*}$ and $b_{i j}=b_{j i}$. Since $S_{m}\left(b_{i j}\right)=R_{3 m}\left(\mathscr{A}_{i j}\right)$, the result follows from Prop. I.2.10.

Imitating the proof of Theorem I.2.9, we have:

THEOREM 1.2.12. If char. $k \neq 3$, the ring $R$ of theta functions is a finitely generated k-algebra.

Finally, by Theorem I.2.9 and Theorem I.2.12, $R$ is a finitely generated $k$-algebra no matter what the characteristic of the field $k$ is.

\section{§ I.3. The structure of Proj. $(R)$}

Let $R$ be the graded ring of theta functions associated with the matrix $\left(\mathscr{A}_{i j}\right)$, let $A$ denote the scheme Proj. $(R)$ and $A_{k}$ the set of its $k$-valued points. Let $\Gamma$ be the multiplicative subgroup of $G_{g}$ generated by the column vectors of $\left(\mathscr{A}_{i j}\right)$. In this section we show that $A$ is an abelian variety of dimension $g$ over $k$ and construct a canonical homomorphism $\varphi: G_{g} / \Gamma \rightarrow A_{k}$.

Let $x$ be any element of $G_{g}$. By Lemma I.2.1, there is a $\theta \in R_{1}$ such that $\theta(x) \neq 0$. Thus we have an evaluation homomorphism $\varphi_{x}: R_{\theta} \rightarrow k$ which induces a morphism $\varphi_{x}$ : Spec. $(k) \rightarrow$ Spec. $\left(R_{\theta}\right)$. This gives us a $k$-valued point $P_{x}$ of $A . P_{x}$ depends only on the class of $x$ modulo $\Gamma$, and we have defined a function: 


$$
\begin{aligned}
\varphi: G_{g} / \Gamma & \rightarrow A_{k} \\
x & \rightarrow P_{x} .
\end{aligned}
$$

The following standard facts will be needed later on.

LEMMA I.3.1. Let $N \subset M$ be graded rings with $M$ integral over $N$. Then the open sets Spec. $\left(M_{n}\right), n \in N_{i}, i>0$, cover Proj. $(M)$ and the maps Spec. $\left(M_{n}\right) \rightarrow$ Spec. $\left(N_{n}\right)$ piece together to give a morphism Proj. $(M)$ $\rightarrow$ Proj. $(N)$.

LEMma I.3.2. Let $M$ and $N$ be graded algebras and $\varphi_{1}, \varphi_{2}$ : Proj. (M) $\rightarrow$ Proj. $(N)$ morphisms. Suppose further that $\varphi_{1}$ and $\varphi_{2}$ have the same restrictions to Spec. $\left(M_{n}\right)$ for some $n \in M_{r}, r>0$, and $M$ is a domain. Then $\varphi_{1}=\varphi_{2}$.

We are now ready to interpret the results of the last two sections geometrically.

THEOREM I.3.3. Let $\beta: R^{\prime} \rightarrow R^{\prime}$ be the map $\theta(X, Y) \rightarrow \theta\left(X Y, X Y^{-1}\right)$. Then:

(1) $R^{\prime}$ is integral over $\beta\left(R^{\prime}\right)$.

(2) $A^{\prime}=$ Proj. $\left(R^{\prime}\right)$ is the scheme theoretic product $A \times A$ of $A$ with itself over $k$.

(3) $\beta$ induces a morphism $\beta^{*}: A \times A \rightarrow A \times A$.

(4) The map $A_{k} \times A_{k} \rightarrow A_{k} \times A_{k}$ induced by $\beta^{*}$ takes $\left(P_{x}, P_{y}\right)$ to $\left(P_{x y}, P_{x y-1}\right)$.

Proof. Assertions (1) and (2) come from Propositions I.1.5 and I.1.4. Lemma I.3.1 and (1) give a morphism $A^{\prime} \rightarrow A^{\prime}$ induced by $\beta$. Since $A^{\prime}$ identifies with $A \times A$ we get the morphism $\beta^{*}$ of (3), and (4) follows from the definition of $\beta$.

With the notations above let:

1) $m: A \times A \longrightarrow A$ be the morphism $A \times A \stackrel{\beta^{*}}{\longrightarrow} A \times A \stackrel{\pi_{1}}{\longrightarrow} A$ where $\pi_{1}$ is projection on the first factor.

2) $-1_{A}: A \rightarrow A$ be the morphism induced by the automorphism $\theta(X)$ $\rightarrow \theta\left(X^{-1}\right)$ of $R$.

3) $O_{A}: A \longrightarrow A$ be the morphism $A \longrightarrow$ Spec. $(k) \stackrel{e}{\longrightarrow} A$ where $e$ is the $k$-valued point $P_{(1, \ldots, 1)}$.

THEOREM I.3.4. With the operations defined above $A$ is a commuta- 
tive group scheme over $k$. The map $\varphi: G_{g} / \Gamma \rightarrow A_{k}$ constructed at the beginning of this section is a group homomorphism.

Proof (In outline). To show that $A$ is a commutative group scheme we must verify the commutativity of certain diagrams expressing the associative and commutative law, and the existence of a unit and inverse. For example, for associativity we must show that the morphisms $m \circ\left(\mathrm{id}_{A} \times m\right)$ and $m \circ\left(m \times \mathrm{id}_{A}\right)$ from $A \times A \times A \rightarrow A$ are the same. To do this we choose affine open subsets $U$ and $V$ on $A \times A \times A$ and $A$ such that $m \circ(\mathrm{id} \times m)$ and $m \circ(m \times \mathrm{id})$ take $U$ into $V$. An obvious but tedious calculation shows that the two induced maps $\Gamma(V) \rightarrow \Gamma(U)$ coincide and we apply Lemma I.3.2 (for a more detailed proof of a similar result see Theorem I.3.5). Finally, (4) of Theorem I.3.3 shows that $m: A_{k} \times A_{k} \rightarrow A_{k}$ takes $\left(P_{x}, P_{y}\right)$ to $P_{x y}$ : i.e. that $x \rightarrow P_{x}$ is a homomorphism.

THEOREM 1.3.5. For each $n>0$ the map $\alpha_{n}^{*}: A \rightarrow A$ induced by $\alpha_{n}$ is just group scheme multiplication by $n$ (which we will denote by $n_{A}$ ).

Proof. Since $R$ is integral over $\alpha_{n}(R)$, we get a morphism of schemes $\alpha_{n}^{*}: A \rightarrow A$. We show first that if $\theta$ and $\theta^{\prime}$ are in $R_{m}^{\prime}$ then the pull-back of $\theta^{\prime} / \theta \in \Gamma\left((A \times A)_{\theta}\right)$ under $\alpha_{n}^{*} \times$ id is $\theta^{\prime}\left(X^{n}, X\right) / \theta\left(X^{n}, X\right)$, at least on some principal open subset $U$ of $A_{\theta\left(X^{n}, X\right)}$.

To see this, take $\psi \neq 0$ in $R_{m}$. Since $R_{m}^{\prime}=R_{m} \otimes R_{m}$, direct calculation shows that the pull-back of $\theta / \psi(X) \psi(Y)$ under $\alpha_{n}^{*} \times$ id. is $\theta\left(X^{n}, X\right)$ $/ \psi\left(X^{n}\right) \psi(X)$. Since a similar formula holds for the pull-back of $\theta^{\prime} / \psi(X) \psi(Y)$, we get our result where $U$ is defined by $\psi\left(X^{n}\right) \psi(X)$.

The theorem can now be proved by induction on $n$. $n=1$ is obvious. $(n+1)_{A}$ is the composite map

$$
\pi_{1} \circ \beta^{*} \circ\left(n_{A} \times \text { id. }\right): A \rightarrow A \times A \rightarrow A \times A \rightarrow A .
$$

Fix $G \neq 0$ in $R_{1}$ and suppose $F \in R_{m}$. Then $F / G^{m}$ in $\Gamma\left(A_{G}\right)$ pulls back to $F(X) G(Y)^{m} / G(X)^{m} G(Y)^{m}$ under $\pi_{1}$ and this pulls back to $F(X Y) G\left(X Y^{-1}\right)^{m}$ $/ G(X Y)^{m} G\left(X Y^{-1}\right)^{m}$ under $\beta^{*}$. By induction, $\left(n_{A} \times \mathrm{id}\right)=\left(\alpha_{n}^{*} \times \mathrm{id}\right)$. If we apply the result of the paragraph above with $\psi=G^{2 m}$, we conclude that the pull-back of $F / G^{m}$ under $(n+1)_{A}=\pi_{1} \circ \beta^{*} \circ\left(\alpha_{n}^{*} \times\right.$ id. $)$ is $F\left(X^{n+1}\right) / G\left(X^{n+1}\right)^{m}$ over the affine subset of $A$ defined by $G\left(X^{n+1}\right) G\left(X^{n-1}\right) G\left(X^{n}\right) G(X)$. The theorem then follows from Lemma I.3.2 applied to the maps $\alpha_{n+1}$ and $(n+1)_{A}$. 
THEOREM I.3.6. The scheme $A=$ Proj. $(R)$ is an abelian variety of dimension $g$ over $k$.

Proof. From Theorem I.3.4, $A$ has the structure of commutative group scheme over $k$. Since $R$ is a finitely generated $k$-algebra and an integral domain, $A$ is of finite type, reduced and irreducible. If $L$ is a finite extension of $k$, let $R(L)$ be the graded $L$-algebra corresponding to the matrix $\left(\mathscr{A}_{i j}\right)$ over the field $L$. Then $R \otimes_{k} L \simeq R(L)$ and is a domain. Hence, $A$ remains reduced and irreducible under finite extensions of $k$, and since it is projective, it is an abelian variety. Since dim. $R_{m}=(2 m)^{g}$ for all $m>0, A$ has dimension $g$.

\section{II}

In this part we show that the map $\varphi: G_{g} / \Gamma \rightarrow A_{k}$ defined in $\S I .3$ is an isomorphism provided the elements off the main diagonal of the matrix $\left(\mathscr{A}_{i j}\right)$ are units in the valuation ring $\mathcal{O}$. Throughout part II we make this assumption on the $\mathscr{A}_{i j}$ 's. Note that $q_{i}=\mathscr{A}_{i i} \in \mathscr{M}$ because of positive definiteness.

\section{§I.1. The reduction of $A$}

Let $R=\oplus_{0}^{\infty} R_{m}$ be the graded ring of theta functions associated to the matrix $\left(\mathscr{A}_{i j}\right)$. If $m$ is a positive integer, let $R_{m, \bullet}$ denote the subspace of $R_{m}$ consisting of Laurent series with coefficients in $\mathcal{O}$. The $2 m$ parity decomposition $R_{m}=\oplus_{(r)} R_{m}^{(r)}, r_{j} \in Z / 2 m Z$, induces a decomposition $R_{m, o}=\oplus_{(r)} R_{m, o}^{(r)}$ where $R_{m, o}^{(r)}=R_{m}^{(r)} \cap R_{m, o}$. Let $\bar{R}_{m}=R_{m, o} / \mathscr{M} R_{m, o}, \bar{R}=\oplus_{0}^{\infty} \bar{R}_{m}$. Then $\bar{R}_{m}$ is a direct sum of 1-dimensional subspaces $\bar{R}_{m}^{(r)}=R_{m, 0}^{(r)} / \mathscr{M} R_{m, 0}^{(r)}$ over $\bar{k}$.

There is an obvious map $R_{m, o} \rightarrow \bar{k}\left[X_{i}, X_{i}^{-1}\right]$ given by $\sum \mathscr{A}_{I} X^{I} \rightarrow \sum \overline{\mathscr{A}}_{I} X^{I}$. The kernel is evidently $\mathscr{M} \cdot R_{m, \boldsymbol{c}}$, so $\bar{R}_{m}$ identifies with a subspace of $\bar{k}\left[X_{i}, X_{i}^{-1}\right]$. We now calculate what this subspace is. Rather than taking $r_{j}$ to be elements of $Z / 2 m Z$ we shall take $r_{j}$ to be integers with $-m$ $<r_{j} \leq m$. Then, by Theorem I.1.1, every $\theta \in R_{m, \theta}$ may be written as $\sum b_{I} X^{I}$ where

$$
b_{I}=\prod_{j=1}^{g} q_{j}^{t_{j}\left(m t_{j}+r_{j}\right)} \prod_{j>\ell} \mathscr{A}_{j \ell}^{i_{i} t_{j}+r_{j} t_{\ell}} \cdot b_{(r)}
$$

where $b_{(r)} \in \mathcal{O}, I=\left(i_{1}, \cdots, i_{\theta}\right)$ and $i_{j}=2 m t_{j}+r_{j}$. 
Now each $\mathscr{A}_{j \ell}(j \neq \ell)$ has order 0 . Also $t_{j}\left(m t_{j}+r_{j}\right) \geq 0$ and equality holds only when $t_{j}=0$ or when $t_{j}=-1$ and $r_{j}=m$. Thus the reduction $\sum \bar{b}_{I} X^{I}$, of $\theta$ only involves monomials with $\left|i_{j}\right| \leq m$. In particular the monomials $X^{I}$ appearing in a generator of $\bar{R}_{m}^{(r)}$ are just those for which the following conditions hold:

$$
\begin{array}{lll}
i_{j}=r_{j} & \text { whenever } & \left|r_{j}\right|<m \\
i_{j}= \pm m & \text { whenever } & r_{j}=m .
\end{array}
$$

Proposition II.1.1. $\quad \bar{R}_{2}$ generates $\bar{R}_{2 m}$ for all $m>0$.

Proof. It suffices to show that $\bar{R}_{1} \bar{R}_{m}=\bar{R}_{m+1}$ for all $m>1$. If $\bar{R}_{m+1}=\oplus_{(r)} \bar{R}_{m+1}^{(r)}$ is the $2(m+1)$-parity decomposition of $\bar{R}_{m+1}$ it suffices to construct a non-zero element of $\bar{R}_{1} \bar{R}_{m}$ of arbitrary $2(m+1)$-parity $(r)=\left(r_{1}, \cdots, r_{g}\right),-(m+1)<r_{j} \leq(m+1)$. We argue by induction on $\sum\left|r_{j}\right|$, and define numbers $c_{j}$ and $d_{j}$ by:

$$
\begin{array}{ll}
c_{j}=0, \quad d_{j}=r_{j} & \text { if }\left|r_{j}\right|<m \\
c_{j}=1 & \text { if } r_{j}=m,-m, m+1 \\
d_{j}=m-1,1-m, m & \text { if } r_{j}=m,-m, m+1 .
\end{array}
$$

Let $\bar{\theta}_{c}$ generate $\bar{R}_{1}^{(c)}$ and $\bar{\theta}_{d}$ generate $\bar{R}_{m}^{(d)}$. The monomials $X^{I}$ appearing in $\bar{\theta}_{c} \bar{\theta}_{d}$ are just those for which:

$$
\begin{array}{lll}
i_{j}=r_{j} & \text { whenever } & \left|r_{j}\right|<m \\
i_{j}=m \text { or } m-2 & \text { whenever } & r_{j}=m \\
i_{j}=-m \text { or } 2-m & \text { whenever } & r_{j}=-m \\
i_{j}= \pm(m+1) \text { or } \pm(m-1) & \text { whenever } & r_{j}=m+1 .
\end{array}
$$

In particular, a generator $\bar{\theta}_{r}$ of $\bar{R}_{m+1}^{(r)}$ occurs as a component of $\bar{\theta}_{c} \bar{\theta}_{d}$. By induction it will suffice to show that every other $\bar{\theta}_{s}$ occurring in $\bar{\theta}_{c} \bar{\theta}_{d}$ has $\sum\left|s_{j}\right|<\sum\left|r_{j}\right|$. Now $X^{s}$ must appear in $\bar{\theta}_{c} \bar{\theta}_{d}$. So by the above, either $s_{j}=r_{j}$, or $\left|r_{j}\right| \geq m$ and $s_{j}= \pm(m-2)$ or $\pm(m-1)$. If $(s) \neq(r)$, we are in this latter case for at least one index $j$. Since $m>1,|m-2|$ $<|m|,\left|s_{j}\right|<\left|r_{j}\right|, \sum\left|s_{j}\right|<\sum\left|r_{j}\right|$ and the proposition is proved.

The above result and Nakayama's Lemma show that $R_{2,0}$ generates $R_{2 m, 0}$ for all $m$. So the graded ring $R_{(2)}=\oplus_{0}^{\infty} R_{2 m}$ is generated by $R_{2}$. Let $\hat{R}_{2}$ be the space of linear maps $R_{2} \rightarrow k$. Then we may identify $A_{k}$ with a Zariski-closed subset of the projectification of $\hat{R}_{2}$. The linear maps $i: R_{2} \rightarrow k$ which correspond to points of $A_{k}$ are those which can 
be extended to $k$-algebra maps $R_{(2)} \rightarrow k$. If $x \in G_{g}$ then $P_{x}$ corresponds to the evaluation map $\theta \rightarrow \theta(x)$.

For $P \in A_{k}$, the corresponding element of $\hat{R}_{2}$ will be denoted by $i_{P}$. We shall normalize $i_{P}$ so that $i_{P}\left(R_{2,0}\right)=\mathcal{O}$. It is still, of course, only determined up to multiplication by a unit of $\mathcal{O}$.

We next define bases $\theta_{\alpha}$ and $\lambda_{\alpha}$, of $R_{2,0}$ and $R_{1, o}$, that we shall make constant use of. Namely, if $\alpha_{j} \in\{-1,0,1,2\}$ let $\theta_{\alpha}$ be a generator of $R_{2,0}^{(\alpha)}$. If $\alpha_{j} \in\{0,1\}$, let $\lambda_{\alpha}$ be a generator of $R_{1,0}^{(\alpha)}$. The monomials $X^{I}$ appearing in $\bar{\theta}_{\alpha}$ are just those for which:

$$
\begin{array}{llll}
i_{j}=\alpha_{j} & \text { whenever } & \alpha_{j}=0,1 \text { or }-1 \\
i_{j}= \pm 2 & \text { whenever } & \alpha_{j}=2 . &
\end{array}
$$

The monomials $X^{I}$ appearing in $\bar{\lambda}_{\alpha}$ are just those for which:

$$
\begin{array}{lll}
i_{j}=0 & \text { whenever } & \alpha_{j}=0 \\
i_{j}= \pm 1 & \text { whenever } & \alpha_{j}=1 .
\end{array}
$$

If $P \in A_{k}$, let $X_{\alpha}(P)=i_{P}\left(\theta_{\alpha}\right)$. The $X_{\alpha}(P)$ are projective coordinates for $P$. Since the $\theta_{\alpha}$ are a basis for $R_{2,0}$ and $i_{P}$ is normalized, the $X_{\alpha}(P)$ are in $\mathcal{O}$, but not all in $\mathscr{M}$.

Now let $\bar{A}=$ Proj. $(\bar{R})$ and $\bar{A}_{\bar{k}}$ be the set of $\bar{k}$-valued points of $\bar{A}$. Since $\bar{R}_{2}$ generates $\bar{R}_{(2)}$ we may identify $\bar{A}_{\bar{k}}$ with a Zariski-closed subset of the projectification of $\hat{R}_{2}$. Let $i_{\bar{P}}$ be the map corresponding to $\bar{P}$. For $\bar{P} \in \bar{A}_{\bar{k}}, X_{\alpha}(\bar{P})=i_{\bar{P}}\left(\bar{\theta}_{\alpha}\right)$ give projective coordinates for $\bar{P}$.

Each normalized $i_{p}: R_{2} \rightarrow k$ gives by reduction a non-zero map $\bar{R}_{2} \rightarrow \bar{k}$. Thus we get a reduction mapping $P \rightarrow \bar{P}$ from $A_{k}$ to $\bar{A}_{\bar{k}}$. If $P$ has projective coordinates $\left\{X_{\alpha}(P)\right\}$, those of $\bar{P}$ are $\left\{\overline{X_{\alpha}(P)}\right\}$.

\section{§II.2. A stratification on $A$}

To simplify notation let $\theta_{0}=\theta_{0, \ldots, 0}$ and $\theta_{j}=\theta_{0, \ldots,(j)}, \ldots, 0$ for $j=1,2$, $\cdots, g$. We may assume that the reductions of $\theta_{0}, \theta_{1}, \theta_{2}, \cdots, \theta_{g}$ are $1, X_{1}, X_{2}$, $\cdots, X_{g}$ respectively. Let $x_{j}$ denote the rational function $\bar{\theta}_{j} / \bar{\theta}_{0} j=1,2$, $\cdots, g$ on $\bar{A}$. Since $\bar{\theta}_{\alpha}$ is a polynomial in $X_{i}$ and $X_{i}^{-1}$ with coefficients in $\bar{k}$, the rational function $\bar{\theta}_{\alpha} / \bar{\theta}_{0}$ on $\bar{A}$ is given by $\sum c_{I} x^{I}, c_{I} \in \bar{k}^{*}$ where the sum extends over all $\left(i_{1}, \cdots, i_{g}\right)$ such that

$$
\begin{array}{lll}
i_{j}=\alpha_{j} & \text { if } & \alpha_{j}=0,1, \text { or }-1 \\
i_{j}= \pm 2 & \text { if } & \alpha_{j}=2 .
\end{array}
$$


THEOREM II.2.1. For each $\bar{P} \in \bar{A}_{\bar{k}}$ there is a unique subset $S=S(\bar{P})$ of $\{1,2, \cdots, g\}$ such that:

$$
\begin{array}{lll}
\text { if } \quad \alpha^{-1}(2)=S, \text { then } & X_{\alpha}(\bar{P}) \neq 0 \\
\text { if } \quad \alpha^{-1}(2) \not \supset S, \text { then } & X_{\alpha}(\bar{P})=0 .
\end{array}
$$

Proof. The uniqueness of $S(\bar{P})$ is obvious. To prove the existence, let $\left(\mathcal{O}_{v}, \mathscr{M}_{v}\right)$ be a valuation ring dominating the local ring $\left(\mathcal{O}_{\bar{P}}, \mathscr{M}_{\bar{P}}\right)$ of $\bar{P}$ on $\bar{A}$. Let $v$ be the order function attached to the ring $\mathcal{O}_{v}$.

With $x_{j}=\bar{\theta}_{j} / \bar{\theta}_{0}$, let $S=\left\{j: v\left(x_{j}\right) \neq 0\right\}$. Writing $\bar{\theta}_{\alpha} / \bar{\theta}_{0}$ as $\sum c_{I} x^{I}$ with $c_{I} \in \bar{k}^{*}$ we see:

$$
\begin{aligned}
& v\left(c_{I} x^{I}\right)=\sum_{j=1}^{g} i_{j} v\left(x_{j}\right) \geq \sum_{j \in S} 2 \min .\left(v\left(x_{j}\right), v\left(x_{j}^{-1}\right)\right) \\
& v\left(\bar{\theta}_{\alpha} / \bar{\theta}_{0}\right) \geq \sum_{j \in S} 2 \min .\left(v\left(x_{j}\right), v\left(x_{j}^{-1}\right)\right) .
\end{aligned}
$$

If $\alpha^{-1}(2)=S$, there is exactly one term $x^{I}$ such that the equality in $\left(^{*}\right)$ holds, so strict equality holds in $\left(^{* *}\right)$. Suppose now that for some $\alpha$ with $\alpha^{-1}(2)=S, X_{\alpha}(\bar{P})=0$. Let $\beta=\left(\beta_{1}, \cdots, \beta_{g}\right)$ be such that $X_{\beta}(\bar{P}) \neq 0$. Then the rational function $\bar{\theta}_{\alpha} / \bar{\theta}_{\beta}$ is in $\mathscr{M}_{\bar{P}} \subset \mathscr{M}_{v}$. Since $\alpha^{-1}(2)=S$, the above calculation shows that:

$$
v\left(\bar{\theta}_{\alpha} / \bar{\theta}_{\beta}\right)=v\left(\bar{\theta}_{\alpha} / \bar{\theta}_{0}\right)-v\left(\bar{\theta}_{\beta} / \bar{\theta}_{0}\right) \leq 0
$$

which is a contradiction, and (1) follows.

In order to prove (2), note that if $\alpha^{-1}(2) \not \supset S$, we have strict inequality in $(* *)$. Now let $\beta$ be such that $\beta^{-1}(2)=S$. By $(1), X_{\beta}(\bar{P}) \neq 0$ and so the rational function $\bar{\theta}_{\alpha} / \bar{\theta}_{\beta} \in \mathcal{O}_{\bar{P}}$. Since $\alpha^{-1}(2) \not \supset S$, the above calculation shows that $v\left(\bar{\theta}_{\alpha} / \bar{\theta}_{\beta}\right)>0$ and so $\bar{\theta}_{\alpha} / \bar{\theta}_{\beta} \in \mathscr{M}_{v}$. Therefore $\bar{\theta}_{\alpha} / \bar{\theta}_{\beta} \in \mathscr{M}_{\bar{P}}$ $=\mathscr{M}_{v} \cap \mathcal{O}_{\bar{P}}$ and (2) follows.

THEOREM II.2.2. Let $i_{\bar{P}}: \bar{R}_{2} \rightarrow \bar{k}$ be the map associated to $\bar{P} \in \bar{A}_{\bar{k}}$ and let $S=S(\bar{P})$. Then:

$$
\begin{aligned}
& \alpha^{-1}(1)=S \Rightarrow i_{\bar{P}}\left(\bar{\lambda}_{\alpha}^{2}\right) \neq 0 \\
& \alpha^{-1}(1) \downarrow S \Rightarrow i_{\bar{P}}\left(\bar{\lambda}_{\alpha}^{2}\right)=0 .
\end{aligned}
$$

Proof. $\bar{\lambda}_{\alpha}^{2} / \bar{\theta}_{0}=\left(\sum d_{I} x^{I}\right)^{2}, d_{I} \in \bar{k}^{*}$ with $i_{j}=0$ when $\alpha_{j}=0, i_{j}= \pm 1$ when $\alpha_{j}=1$, and $x_{j}=\bar{\theta}_{j} / \bar{\theta}_{0}$.

It follows that:

$$
v\left(\bar{\lambda}_{\alpha}^{2} / \bar{\theta}_{0}\right) \geq \sum_{j \in S} 2 \min .\left(v\left(x_{j}\right), v\left(x_{j}^{-1}\right)\right)
$$


with equality if $\alpha^{-1}(1)=S$ and strict inequality if $\alpha^{-1}(1) \ngtr S$.

To prove (1) suppose $\alpha_{i} \in\{0,1\}$ with $\alpha^{-1}(1)=S$. Choose $\beta_{i} \in\{-1,0$, $1,2\}$ so that $\beta^{-1}(2)=S$. By Th. II.2.1, $\bar{\lambda}_{\alpha}^{2} / \bar{\theta}_{\beta} \in \mathcal{O}_{\bar{P}}$. Furthermore:

$$
v\left(\bar{\lambda}_{\alpha}^{2} / \vec{\theta}_{\beta}\right)=v\left(\bar{\lambda}_{\alpha}^{2} / \bar{\theta}_{0}\right)-v\left(\bar{\theta}_{\beta} / \bar{\theta}_{0}\right)=0 .
$$

Thus $\bar{\lambda}_{\alpha}^{2} / \bar{\theta}_{\beta}$ is a unit in $\mathcal{O}_{\bar{P}}$ and (1) follows.

Similarly, if $\alpha^{-1}(1) \not \supset S, \bar{\lambda}_{\alpha}^{2} / \bar{\theta}_{\beta} \in \mathcal{O}_{\bar{P}} \cap \mathscr{M}_{v}=\mathscr{M}_{\bar{P}}$ and (2) follows.

Suppose now $P \in A_{k}$ with reduction $\bar{P}$. By the support $S(P)$ of $P$ we mean the set $S(\bar{P})$ of Theorem II.2.1. We conclude this section with some remarks which we will use constantly.

(a) $P \in A_{k}$ has empty support if and only if $X_{0}(P)$ is a unit.

(b) Suppose $y=\left(y_{1}, \cdots, y_{g}\right) \in G_{g}$ with $\mid$ ord. $y_{j} \mid \leq \frac{1}{2}$ ord. $q_{j}$. Then $S(\varphi(y))$ $=\left\{j:\right.$ ord. $\left.y_{j} \neq 0\right\}$

(c) $\lambda_{\alpha}(X)=\sum b_{I} X^{I}$ where $i_{j}=2 t_{j}+\alpha_{j}$ and ord. $b_{I}=\sum_{j} t_{j}\left(t_{j}+\alpha_{j}\right)$ ord. $q_{j}$

(d) $\theta_{\alpha}(X)=\sum b_{I} X^{I}$ where $i_{j}=4 t_{j}+\alpha_{j}$ and ord. $b_{I}=\sum_{j} t_{j}\left(2 t_{j}+\alpha_{j}\right)$ ord. $q_{j}$.

(a) is immediate from the definitions of $S(P)$. We call such points unit points; in the next section we study them carefully. We get (c) and (d) by specifying $m$ to be 1 or 2 in the remarks before Prop. II.1.1. To prove (b) we use:

LEMMA II.2.3. Let $0 \neq q \in \mathscr{M}$ and $y \in k^{*}$ with |ord. $y \mid \leq \frac{1}{2}$ ord. $q$. Let $\alpha \in\{0,1\}, t \in Z$ and set $s=t(t+\alpha)$ ord. $q+(2 t+\alpha)$ ord. $y$. Then:

(1) if $\alpha=0, s \geq 0$

(2) if $\alpha=1, s \geq-\mid$ ord. $y \mid$. For ord. $y>0$ (respectively ord. $y<0$ ) equality occurs if and only if $t=-1$ (respectively $t=0$ ).

Proof. (1) is trivial. In order to prove (2) note that if ord. $y \geq 0$ then $s \geq\left(2(t+1)^{2}-1\right)$ ord. $y$, and if ord. $y<0, s \geq\left(2 t^{2}-1\right) \mid$ ord. $y \mid$.

LEMMA II.2.4. Suppose $y=\left(y_{1}, \cdots, y_{g}\right) \in G_{g}$ with $\mid$ ord. $y_{j} \mid \leq \frac{1}{2}$ ord. $q_{j}$. Let $S=\left\{j\right.$ : ord. $\left.y_{j} \neq 0\right\}$. Suppose $\alpha_{j} \in\{0,1\}$. Then: ord. $\lambda_{\alpha}(y) \geq-\sum_{j \in S} \mid$ ord. $y_{j} \mid$.

Furthermore, equality holds if $\alpha^{-1}(1)=S$ and inequality holds if $\alpha^{-1}(1) \not \supset S$.

Proof. By $(c), \lambda_{\alpha}(y)=\sum b_{I} y^{I}$ where 
ord. $\left(b_{I} y^{I}\right)=\sum_{j=1}^{g} s_{j}=\sum_{j=1}^{g} t_{j}\left(t_{j}+\alpha_{j}\right)$ ord. $q_{j}+\left(2 t_{j}+\alpha_{j}\right)$ ord. $y_{j}$.

So by Lemma II.2.3, ord. $\left(b_{I} y^{I}\right) \geq-\sum_{j \in S} \mid$ ord. $y_{j} \mid$ giving $\left(^{*}\right)$. Suppose now that $\alpha^{-1}(1)=S$. Then there is precisely one monomial $b_{I} y^{I}$ in $\lambda_{\alpha}(y)$ such that ord. $\left(b_{I} y^{I}\right)=-\sum_{j \in S} \mid$ ord. $y_{j} \mid$ (whenever ord. $y_{j}=0, t_{j}=0$. When ord. $y_{j}>0, t_{j}=-1$ and when ord. $y_{j}<0, t_{j}=0$ ). Thus equality holds in (*). Finally, if $\alpha^{-1}(1) \not \supset S$, there is an index $j$ such that $\alpha_{j}=0$ and ord. $y_{j} \neq 0$. Then, $s_{j} \geq 0>-\mid$ ord. $y_{j} \mid$ and the last assertion follows.

Remark (b) is an immediate consequence of Lemma II.2.4 and Theorem II.2.2. (note that $i_{P}\left(\lambda_{\alpha}^{2}\right)=\lambda_{\alpha}(y)^{2}$ up to multiplication by a nonzero constant independent of $\alpha$ ).

\section{§II.3. The unit points of $A_{k}$}

Let $U$ denote the multiplicative group of units of the ring $\mathcal{O}$ and $U_{k}$ be the set of unit points of $A_{k}$ (i.e. points with empty support). In this section we show that $\varphi$ induces a bijection $U^{g} \rightarrow U_{k}$. The injectivity of $\varphi: G_{g} / \Gamma \rightarrow A_{k}$ follows easily.

Let $P \in U_{k}$. We shall normalize the coordinates of $P$ so that $X_{0}(P)$ $=1$. Then $X_{\alpha}(P) \in \mathcal{O}$ for all $\alpha:\{1,2, \cdots, g\} \rightarrow\{-1,0,1,2\}$. Furthermore, if $P \in A_{k}$ and $\alpha$ is such that $\alpha^{-1}(2)=\varnothing$, then $X_{\alpha}(P) \in U$. In particular $X_{1}(P), \cdots, X_{g}(P)$ are in $U$. (here $\left.X_{j}=X_{0, \cdots, \frac{1}{(j)}, \cdots, 0}\right)$.

THEOREM II.3.1. The restriction of the canonical map $\varphi: G_{g} / \Gamma \rightarrow A_{k}$ to $U^{g}$ is a bijection of $U^{g}$ with $U_{k}$.

Proof. If $x \in U^{g}$, it follows from remark (b) of $\S$ II.2. that $\varphi(x) \in U_{k}$. In order to prove bijectivity, it is enough to show the following:

(1) $\psi: U^{g} \rightarrow U^{g} ; x \rightarrow\left(\theta_{1}(x) / \theta_{0}(x), \cdots, \theta_{g}(x) / \theta_{0}(x)\right)$ is $1-1$ and onto.

(2) Two unit points with the same values of $X_{1}, \cdots, X_{g}$ must be equal.

We proceed to prove (1) and (2). We may normalize the $\theta_{i}$ so that $\theta_{0}=1+\cdots$, and $\theta_{j}=X_{j}+\cdots$. Then $\psi$ is "close to the identity" so (1) is intuitively clear. To give a rigorous proof, suppose $u=\left(u_{1}, \cdots, u_{g}\right)$ $\in U^{g}$. Let $T: U^{g} \rightarrow U^{g}$ be the map $x \rightarrow x-\psi(x)+u$. It suffices to show that $T$ has a unique fixed point.

Let $r=\min$. (ord. $\left.q_{j}\right)$. If $x, y \in U^{g}$ set ord. $(x-y)=\min$. ord. $\left(x_{j}-y_{j}\right)$. We know that $\theta_{0}(X)=\sum C_{I} X^{I}$ where $i_{j}=4 t_{j}$ and ord. $C_{I}=\sum 2 t_{j}^{2}$ ord. $q_{j}$. So if $I \neq(0, \cdots, 0)$, ord. $C_{I} \geq r$. It follows that if $x, y \in U^{g}$ : 
(a)

ord. $\left(\theta_{0}(x)-\theta_{0}(y)\right) \geq \operatorname{ord} .(x-y)+r$.

Let $\theta_{j}^{*}(X)=\theta_{j}(X)-X_{j} \theta_{0}(X)$. A similar calculation gives:

$$
\text { ord. }\left(\theta_{j}^{*}(x)-\theta_{j}^{*}(y)\right) \geq \operatorname{ord} .(x-y)+r .
$$

Now the difference between the $j^{\prime}$ th coordinate of $T(x)$ and of $T(y)$ is $\theta_{j}^{*}(x) / \theta_{0}(x)-\theta_{j}^{*}(y) / \theta_{0}(y)$. Using (a), (b) and the fact that $\theta_{0}(x)$ and $\theta_{0}(y)$ are units, we see that this has ord. $\geq$ ord. $(x-y)+r$. So $T$ is a contraction mapping. Since $k$ is complete, so is $U^{g}$, and $T$ has a unique fixed point.

To prove (2) note that for any $\alpha,\left(\bar{\theta}_{0}\right)^{2 g-1}\left(\prod_{i=1}^{g}\left(\bar{\theta}_{i}\right)^{2}\right) \bar{\theta}_{\alpha}$ is an element of $\bar{R}_{8 g}$ which only contains terms $X^{I}$ with $0 \leq i_{j} \leq 4$. So we may write:

$$
\left(\bar{\theta}_{0}\right)^{2 g-1}\left(\prod_{i=1}^{g}\left(\bar{\theta}_{i}\right)^{2}\right) \bar{\theta}_{\alpha}=\bar{F}_{\alpha}\left(\bar{\theta}_{0}, \bar{\theta}_{1}, \cdots, \bar{\theta}_{g}\right)
$$

where $\bar{F}_{\alpha}$ is a homogeneous polynomial of degree $4 g$ with coefficients in $\bar{k}$. Lift $\bar{F}_{\alpha}$ to a homogeneous $F_{\alpha}$ with coefficients in $\mathcal{O}$. Then $\theta_{\alpha}^{2 g-1}\left(\prod_{i=1}^{g} \theta_{i}^{2}\right) \theta_{\alpha}$ and $F_{\alpha}\left(\theta_{0}, \theta_{1}, \cdots, \theta_{g}\right)$ differ by an element of $\mathscr{M} R_{8 g, \bullet}$. Since $R_{2, \bullet}$ generates $R_{8 g, 0}$ we have:

$$
\theta_{0}^{2 g-1}\left(\prod_{i=1}^{g} \theta_{i}^{2}\right) \theta_{\alpha}=F_{\alpha}\left(\theta_{0}, \theta_{1}, \cdots, \theta_{g}\right)+C G_{\alpha}\left(\theta_{\beta}\right)
$$

where $C \in \mathscr{M}$ and may be taken independent of $\alpha$, and each $G_{\alpha}$ has coefficients in $\mathcal{O}$. From this we deduce polynomial identities that hold on all A. Namely suppose $P \in A_{k}$ with $X_{0}(P)=1$. Then:

$$
\left(\prod_{i=1}^{g} X_{i}(P)^{2}\right) X_{\alpha}(P)=f_{\alpha}\left(X_{1}(P), \cdots, X_{g}(P)\right)+C g_{\alpha}\left(X_{\beta}(P)\right)
$$

where $f_{\alpha}, g_{\alpha}$ have coefficients in $\mathcal{O}$. Suppose now that $P$ and $Q$ are unit points with $X_{j}(P)=X_{j}(Q)$. Then $X_{\alpha}(P)$ and $X_{\alpha}(Q)$ are in $\mathcal{O}$ and each $X_{j}(P)$ is a unit. $\left(^{*}\right)$ and an easy induction show that $X_{\alpha}(P) \equiv X_{\alpha}(Q) \bmod C^{n}$ for all $n$. So $X_{\alpha}(P)=X_{\alpha}(Q)$ and $P=Q$.

THEOREM II.3.2. $\varphi: G_{g} / \Gamma \rightarrow A_{k}$ is injective.

Proof. Suppose $\varphi(x)=\varphi(1)=P$. Modifying $x$ by an element of $\Gamma$ we may assume $x=\left(x_{1}, \cdots, x_{g}\right)$ with $\mid$ ord. $x_{j} \mid \leq \frac{1}{2}$ ord. $q_{j}$. Now $P$ is a unit point. So by remark (b) of $\S$ II. 2 each ord. $x_{j}=0$ and $x \in U^{g}$. By the theorem above, $x=1$. 


\section{§ II.4. An addition formula}

THEOREM II.4.1. Suppose $Q, R \in A_{k}$ with disjoint supports. Then $S(Q R)=S(Q) \cup S(R)$.

The proof of this result will occupy the rest of this section. It is based on an addition formula, Theorem II.4.6, which plays a central role in this paper. Recall that $A^{\prime}$ is the abelian variety attached to the $2 g \times 2 g$ matrix with two copies of $\left(\mathscr{A}_{i j}\right)$ down its diagonal and ones elsewhere. We identify $\{1,2, \cdots, 2 g\}$ with the disjoint union of two copies of $\{1,2, \cdots, g\}$ in the obvious way. Then a map $\alpha:\{1,2, \cdots, 2 g\}$ $\rightarrow\{0,1\}$ may be thought of as a pair of maps $\beta$ and $\gamma:\{1,2, \cdots, g\} \rightarrow\{0,1\}$. Under the identification of $R_{1}^{\prime}$ with $R_{1} \otimes R_{1}, \lambda_{\alpha}(X, Y)$ corresponds to $\lambda_{\beta}(X) \lambda_{r}(Y)$, and similarly for $R_{2}^{\prime}$ and $\theta_{\alpha}(X, Y)$. If $P \in A_{k}^{\prime}, S(P)$ may be thought of as a subset of the disjoint union of two copies of $\{1,2, \cdots, g\}$. On the other hand $P$ identifies with some $(Q, R) \in A_{k} \times A_{k}$ and we have:

LEMMA II.4.2. $S(P)$ is the disjoint union of $S(Q)$ in the first copy of $\{1,2, \cdots, g\}$ and $S(R)$ in the second.

Proof. $i_{P}\left(\lambda_{\beta, r}(X, Y)\right)=i_{Q}\left(\lambda_{\beta}\right) i_{R}\left(\lambda_{r}\right)$. The result follows easily from Theorem II.2.2. applied to $A^{\prime}$.

LEMma II.4.3. Let $Q, R \in A_{k}$. Suppose there is a subset $S$ of $\{1,2$, $\cdots, g$ s such that

$$
\text { ord. } i_{(Q, R)}\left(\lambda_{\beta}(X Y) \lambda_{r}\left(X Y^{-1}\right)\right) \geq 0
$$

for all $\beta, \gamma:\{1,2, \cdots, g\} \rightarrow\{0,1\}$, with equality if $\beta^{-1}(1)=\gamma^{-1}(1)=S$ and inequality if $\beta^{-1}(1) \not \supset S$ or $\gamma^{-1}(1) \not \supset S$. Then $S(Q R)=S$.

Proof. $i_{\left(Q R, Q R^{-1)}\right.}\left(\lambda_{\beta}(X) \lambda_{\gamma}(Y)\right)=i_{(Q, R)}\left(\lambda_{\beta}(X Y) \lambda_{\gamma}\left(X Y^{-1}\right)\right)$. So if the hypotheses of the lemma hold, Theorem II.2.2 applied to $A^{\prime}$ shows that the support of $\left(Q R, Q R^{-1}\right)$ is the disjoint union of two copies of $S$. By Lemma II.4.2, $S(Q R)=S\left(Q R^{-1}\right)=S$.

LEMMA II.4.4. Suppose the monomial $X^{\delta} Y^{\eta}$ appears in $\lambda_{\beta}(X Y)$ $\cdot \lambda_{y}\left(X Y^{-1}\right)$. Then:

$(*) \quad \int \begin{aligned} & \text { whenever } \beta_{j}=\gamma_{j} \text { both } \delta_{j} \text { and } \eta_{j} \text { are even } \\ & \text { whenever } \beta_{j} \neq \gamma_{j} \text { both } \delta_{j} \text { and } \eta_{j} \text { are odd }\end{aligned}$ 


$$
\left\{\begin{array}{l}
\text { whenever } \quad \gamma_{j}=0, \delta_{j} \equiv \eta_{j} \bmod .4 \\
\text { whenever } \quad \gamma_{j}=1, \delta_{j} \neq \equiv \eta_{j} \bmod .4
\end{array}\right.
$$

Proof. $\quad \lambda_{\beta}(X Y) \lambda_{r}\left(X Y^{-1}\right)$ is a sum of monomials of the form $X^{m+n} Y^{m-n}$ with $m_{j} \equiv \beta_{j} \bmod .2$ and $n_{j} \equiv \gamma_{j} \bmod .2$. The result follows.

LEMMA II.4.5. Suppose we are given $\beta_{j}, \gamma_{j}, \delta_{j}, \eta_{j}$ such that $\beta_{j}$ and $\gamma_{j}$ are in $\{0,1\}, \delta_{j}$ and $\eta_{j}$ are in $\{0, \pm 1,2\}$, and $\left(^{*}\right)$ of Lemma II.4.4 is satisfied. Then the coefficient of $X^{\delta} Y^{\eta}$ in $\lambda_{\beta}(X Y) \lambda_{r}\left(X Y^{-1}\right)$ is (unit) $\left(\prod_{j} q_{j}\right)$ where $j$ runs over all indices such that $\delta_{j}=\eta_{j}=2$.

Proof. Let $\lambda_{\beta}(X)=\sum b_{I} X^{I}$ and $\lambda_{r}(X)=\sum C_{J} X^{J}$. The coefficient we are studying is just $b_{(\delta+\eta) / 2} C_{(\delta-\eta) / 2}$. (*) shows that $\left(\delta_{j}+\eta_{j}\right) / 2 \equiv \beta_{j} \bmod .2$, and that $\left(\delta_{j}-\eta_{j}\right) / 2 \equiv \gamma_{j} \bmod .2$. Also $\left(\delta_{j}+\eta_{j}\right) / 2$ and $\left(\delta_{j}-\eta_{j}\right) / 2$ are both in $\{0, \pm 1\}$ except for the single exceptional case $\delta_{j}=\eta_{j}=\left(\delta_{j}+\eta_{j}\right) / 2=2$. The result now follows from remark (c) of $\S$ II.2.

THEOREM II.4.6. $\quad \lambda_{\beta}(X Y) \lambda_{\gamma}\left(X Y^{-1}\right)=\sum_{\delta, \eta} C_{\delta, \eta} \theta_{\delta}(X) \theta_{\eta}(Y)$. Here $\delta$ and $\eta$ range over all maps $\{1, \cdots, g\} \rightarrow\{0, \pm 1,2\}$ satisfying (a) and (b) below, and $C_{\delta, \eta}=($ unit $)\left(\prod q_{j}\right)$, the product ranging over all $j$ such that $\delta_{j}=$ $\eta_{j}=2$.

(a) whenever $\beta_{j}=\gamma_{j}$ then $\delta_{j}$ and $\eta_{j}$ are in $\{0,2\}$. They are equal when $\gamma_{j}=0$ and unequal when $\gamma_{j}=1$.

(b) whenever $\beta_{j} \neq \gamma_{j}$ then $\delta_{j}$ and $\eta_{j}$ are in $\{-1,1\}$. They are equal when $\gamma_{j}=0$ and unequal when $\gamma_{j}=1$

Proof. $\lambda_{\beta}(X Y) \lambda_{r}\left(X Y^{-1}\right) \in R_{2}^{\prime}$ and so may be written as $\sum_{\delta, \eta} C_{\delta, \eta} \theta_{\delta}(X)$ $\cdot \theta_{\eta}(Y)$. Lemma II.4.4 shows that only $\delta$ and $\eta$ satisfying (a) and (b) can occur in this decomposition. Comparing coefficients of $X^{\delta} Y^{\eta}$ and using Lemma II.4.5 we get the result.

Taking every $\beta_{j}$ and $\gamma_{j}$ equal to 1 in Theorem II.4.6 we get:

TheOREM II.4.7.

$$
\lambda_{1, \ldots, 1}(X Y) \lambda_{1, \ldots, 1}\left(X Y^{-1}\right)=\sum_{\alpha} C_{\alpha} \theta_{2-\alpha}(X) \theta_{\alpha}(Y)
$$

where $\alpha$ ranges over all maps $\{1,2, \cdots, g\} \rightarrow\{0,2\}$ and the $C_{\alpha}$ are units.

We now prove Theorem II.4.1. Suppose $S(Q) \cap S(R)=\varnothing$, and let $S=S(Q) \cup S(R)$. It suffices to show that the hypotheses of Lemma II.4.3 are satisfied. So, by Theorem II.4.6 we must show that $\sum C_{\delta, \eta} X_{\delta}(Q) X_{\eta}(R)$ 
is a unit when $\beta^{-1}(1)=\gamma^{-1}(1)=S$ and is in $\mathscr{M}$ when $\beta^{-1}(1) \not \supset S$ or $\gamma^{-1}(1)$ $\not \supset S$.

Suppose first that $\beta^{-1}(1)=\gamma^{-1}(1)=S$. For $j \in S(Q)$ let $\delta_{j}=2$ and $\eta_{j}=0$, for $j \in S(R)$ let $\delta_{j}=0$ and $\eta_{j}=2$ and for $j \notin S$ let $\delta_{j}=\eta_{j}=0$. Then $\delta, \eta$ satisfy the conditions of Theorem II.4.6 and $C_{\hat{\delta}, \eta} X_{\hat{\delta}}(Q) X_{\eta}(R)$ is a unit. Suppose we have any pair $\delta, \eta$ appearing in the expansion of $\lambda_{\beta}(X Y) \lambda_{r}\left(X Y^{-1}\right)$. If $X_{\delta}(Q)$ is to be a unit we must have $\delta_{j}=2$ (and $\eta_{j}=0$ ) for $j \in S(Q)$. If $X_{\eta}(R)$ is a unit, $\eta_{j}=2$ (and $\delta_{j}=0$ ) for $j \in S(R)$. Finally if $C_{\tilde{\delta}, \eta}$ is a unit, $\delta_{j}=\eta_{j}=0$ for $j \notin S$. So $C_{\delta, \eta} X_{\delta}(Q) X_{\eta}(R)$ is a unit for a single pair and $\sum C_{\tilde{\delta}, \eta} X_{\delta}(Q) X_{\eta}(R)$ is a unit.

Suppose next that $\beta^{-1}(1) \not \supset S$. Take an index $j \in S$ such that $\beta_{j}=0$. If $\gamma_{j}=1$ then $\delta_{j}$ and $\eta_{j}$ are in $\{ \pm 1\}$ and $X_{\delta}(Q) X_{\eta}(R) \in \mathscr{M}$. If $\gamma_{j}=0$ then either $\delta_{j}=\eta_{j}=0$ so that $X_{\delta}(Q) X_{\eta}(R) \in \mathscr{M}$, or $\delta_{j}=\eta_{j}=2$ so that $C_{\delta, \eta} \in \mathscr{M}$. Thus $\sum C_{\hat{o}, \eta} X_{\delta}(Q) X_{\eta}(R) \in \mathscr{M}$. We argue similarly if $\gamma^{-1}(1) \not \supset S$.

\section{§I.5. The function $\theta_{P}$}

Let $P \in A_{k}$. Then $i_{P}: R_{2} \rightarrow k$ induces a map $i_{P} \otimes 1: R_{2}^{\prime}=R_{2} \otimes R_{2} \rightarrow R_{2}$. If $\theta \in R_{2}^{\prime}$ its image under $i_{P} \otimes 1$ is denoted by $(\theta \mid X=P)$. If $P=\varphi(x)$, then $(\theta(X, Y) \mid X=P)$ is just the Laurent series $\theta(x, Y)$.

We abbreviate $\lambda_{1, \ldots, 1}$ to $\lambda_{1}$ and let $\psi$ be the element $\lambda_{1}(X Y) \lambda_{1}\left(X Y^{-1}\right)$ of $R_{2}^{\prime}$. For $P \in A_{k}$ let $\theta_{P}=(\psi \mid X=P) . \quad \theta_{P}$, like $i_{P}$, is determined up to multiplication by a unit in $\mathcal{O}$.

If $\theta \in R_{2}$ and $Q \in A_{k}$ we say that $\theta(Q)=0$ if $i_{Q}(\theta)=0$. Note that $\theta(\varphi(x))=0$ if and only if $\theta(x)=0$. We shall need a simple result, Proposition II.5.2, concerning the zeroes of $\theta_{P}$, which follows from:

LEMMA II.5.1. $\theta_{P}(Q)=0$ if and only if either $\lambda_{1}^{2}(P Q)=0$ or $\lambda_{1}^{2}\left(P Q^{-1}\right)$ $=0$.

Proof. $(P, Q)$ and $\left(P Q, P Q^{-1}\right)$ are in $A_{k}^{\prime}=A_{k} \times A_{k}$ and so give homomorphisms $R_{(2)}^{\prime} \rightarrow k$. $i_{(P, Q)}=i_{P} \otimes i_{Q}$ and $i_{(P Q, P Q-1)} \theta(X, Y)=i_{(P, Q)} \theta(X Y$, $\left.X Y^{-1}\right)$.

Thus:

$$
\begin{aligned}
i_{P Q}\left(\lambda_{1}^{2}\right) i_{P Q^{-1}}\left(\lambda_{1}^{2}\right) & =i_{(P Q, P Q-1)}\left(\lambda_{1}(X)^{2} \lambda_{1}(Y)^{2}\right) \\
& =i_{(P, Q)}\left(\psi^{2}\right)=\left(i_{P} \otimes i_{Q}\right)\left(\psi^{2}\right)=i_{Q}\left(\theta_{P}^{2}\right)
\end{aligned}
$$

and the result follows. 
Proposition II.5.2. Suppose $P, Q, R \in A_{k}$ and $\theta_{P}(R)=0$. Then, either $\theta_{P Q-1}(Q R)=0$ or $\theta_{P Q}\left((Q R)^{-1}\right)=0$.

We next study the Laurent expansion of $\theta_{P}$.

Proposition II.5.3. $\theta_{P}=\sum C_{\alpha} X_{2-\alpha}(P) \theta_{\alpha}$ where $\alpha$ ranges over all maps $\{1,2, \cdots, g\} \rightarrow\{0,2\}$ and each $C_{\alpha}$ is a unit.

Proof. Apply $i_{P} \otimes 1$ to both sides of Theorem II.4.7.

Proposition II.5.4. The reduction of $\theta_{P}(Y)$ is a non-zero polynomial in $Y_{j}$ and $Y_{j}^{-1}(1 \leq j \leq g)$, which does not involve $Y_{j}$ or $Y_{j}^{-1}$ if $j \in S(P)$.

Proof. If $\alpha:\{1,2, \cdots, g\} \rightarrow\{0,2\}$ is chosen so that $\alpha^{-1}(0)=S(P)$, then $X_{2-\alpha}(P)$ is a unit. So by Proposition II.5.3 $\bar{\theta}_{P} \neq 0$. Suppose now $j \in S(P)$. Then, if $\alpha_{j}=0, \bar{\theta}_{\alpha}$ does not involve $Y_{j}$ or $Y_{j}^{-1}$ while if $\alpha_{j}=2, X_{2-\alpha}(P)$ $\in \mathscr{M}$. The result follows.

\section{§II.6. The decomposition theorem}

Throughout this section we assume $k$ algebraically closed. Our goal is the following "decomposition theorem": Suppose $P \in A_{k}$. Then $P$ $=Q R$ where $Q=\varphi(z, 1, \cdots, 1)$ for some $z \in k^{*}$ and $1 \notin S(R)$. We begin the proof with a criterion which guarantees that $1 \notin S(R)$. Suppose $R \in A_{k}$ and $\left(\bar{u}_{2}, \cdots, \bar{u}_{g}\right) \in\left(\bar{k}^{*}\right)^{g-1}$. We say that $\left(\bar{u}_{2}, \cdots, \bar{u}_{g}\right)$ is in $\bar{N}_{R}$ if there exists $u=\left(u_{1}, \cdots, u_{g}\right) \in U^{g}$ such that $u_{i}$ lifts $\bar{u}_{i}$ for $i>1$, and $\theta_{R}(u)=0$.

Proposition II.6.1. If $1 \in S(R)$, then $\bar{N}_{R}$ is contained in a proper Zariski-closed subset of $\left(\bar{k}^{*}\right)^{g-1}$.

Proof. Let $\bar{\theta}_{R}$ be the reduction of $\theta_{R}$. By Proposition II.5.4, $\bar{\theta}_{R}$ is a non-zero polynomial in $Y_{j}$ and $Y_{j}^{-1}$ for $j>1$. If $\left(\bar{u}_{2}, \cdots, \bar{u}_{g}\right) \in \bar{N}_{R}$ then $\bar{\theta}_{R}\left(\bar{u}_{2}, \cdots, \bar{u}_{g}\right)=0$.

We next derive some simple results on the zeroes of power series and Laurent series in one variable.

LEMMA II.6.2. Suppose $H(X)=\sum_{0}^{\infty} \mathscr{A}_{i} X^{i} \in \mathcal{O}[[X]]$ with $\bar{H} \neq 0$ and $\mathscr{A}_{0} \in \mathscr{M}$. Then there exists an $x \in \mathscr{M}$ such that $H(x)=0$.

Proof. Let $s$ be the smallest index such that $\mathscr{A}_{s}$ is a unit. By the Weierstrass Preparation Theorem, $H(X)=G \cdot\left(X^{s}-\sum_{0}^{s-1} C_{i} X^{i}\right)$ where $G$ is a unit in $\mathcal{O}[[X]]$ and each $C_{i} \in \mathscr{M}$. Now $k$ is algebraically closed and 
$x$ may be taken to be any root of $X^{s}-\sum_{0}^{s-1} C_{i} X^{i}$.

LEMMA II.6.3. Let $\mathscr{L}_{\circ}$ be the ring of everywhere convergent Laurent series, $\sum_{-\infty}^{\infty} \mathscr{A}_{i} X^{i}$, with $\mathscr{A}_{i} \in \mathcal{O}$. Suppose $G \in \mathscr{L}_{0}$ with $\bar{G} \neq 0$. Then any root $\bar{x}$ of $\bar{G}$ in $\bar{k}^{*}$ lifts to a root of $G$ in $U$.

Proof. Let $x \in U$ be any lifting of $\bar{x}$. Replacing $G$ by $G(x X)$ we may assume $\bar{x}=1$. Let $\psi: \mathscr{L}_{0} \rightarrow \mathcal{O}[[Y]]$ be the homomorphism mapping $X$ on $1-Y$, and $H=\psi(G)$. Then $\bar{H}=\bar{G}(1-Y) \neq 0$, and $\bar{H}(0)=0$. By the lemma above, $H(y)=0$ for some $y \in \mathscr{M}$, and $G(1-y)=0$.

The next result requires some notation. Suppose $G$ is an everywhere convergent Laurent series in $X_{0}, X_{1}, \cdots, X_{n}$ and $u=\left(u_{1}, \cdots, u_{n}\right) \in U^{n}$. Let $G_{u}$ be the 1-variable Laurent series $G\left(X, u_{1}, \cdots, u_{g}\right)$. If $g(X)=\sum b_{I} X^{I}$ is an everywhere convergent Laurent series let ord. $g=\min$. (ord. $b_{I}$ ). Finally if $\bar{g}$ is a polynomial over $\bar{k}$ in $X_{j}$ and $X_{j}^{-1}(1 \leq i \leq n)$ let $\left(U^{n}\right)_{\bar{g}}$ $=\left\{u \in U^{n}: \bar{g}(\bar{u}) \neq 0\right\}$.

LEMMA II.6.4. Suppose $G$ is an everywhere convergent Laurent series in $X_{0}, \cdots, X_{n}$. Write $G=\sum_{-\infty}^{\infty} g_{i}\left(X_{1}, \cdots, X_{n}\right) X_{0}^{i}$ and suppose that for at least two indices $i, g_{i} \neq 0$. Then there exists a real number $r$ and $a \bar{g} \neq 0$ such that whenever $u \in\left(U^{n}\right)_{g}$ there exists a $y \in k^{*}$ with $G_{u}(y)=0$ and ord. $y=r$.

Proof. Let $d_{i}=$ ord. $g_{i}$. We may assume min. $d_{i}=0$. Multiplying $G$ by a power of $X_{0}$ and replacing $X_{0}$ by $X_{0}^{-1}$ if necessary we may assume that $d_{0}=0$ and that $d_{j} \neq \infty$ for some positive $j$. Suppose first that $d_{j}=0$ for some $j>0$. Take $r=0$ and $\bar{g}=\bar{g}_{0} \bar{g}_{j}$. Then if $u \in\left(U^{n}\right)_{\bar{g}}$, $g_{0}(u)$ and $g_{j}(u)$ are units. So $G_{u}=\sum g_{i}(u) X^{i}$ has at least two unit coefficients, $\bar{G}_{u}$ has a root in $\bar{k}^{*}$ and $G_{u}$ has a root with ord. $=0$ by Lemma II.6.3. In general note that $d_{i} / i \rightarrow \infty$ with $i$. Let $r=-\min _{i>0} d_{i} / i$ and choose $C \in k^{*}$ with ord. $C=r$. Replacing $G$ by $G\left(C X_{0}, X_{1}, \cdots, X_{n}\right)$ we reduce to the previously handled case.

We apply the above result to $\theta_{P}$, where $P$ is a given element of $A_{k}$.

Proposition II.6.5. There exists a real number $r$ and an $\vec{h} \neq 0$ such that whenever $\left(u_{2}, \cdots, u_{g}\right) \in U^{g-1}$ with $\vec{h}\left(\bar{u}_{2}, \cdots, \bar{u}_{g}\right) \neq 0$, then there exists a $y \in k^{*}$ with $\theta_{P}\left(y, u_{2}, \cdots, u_{q}\right)=0$ and ord. $y=r$.

Proof. $\theta_{P}=\sum C_{\alpha} X_{2-\alpha}(P) \theta_{\alpha}$ and the $C_{\alpha} X_{2-\alpha}(P)$ do not all vanish. So 
if we write $\theta_{P}(X)=\sum_{-\infty}^{\infty} h_{i}\left(X_{2}, \cdots, X_{g}\right) X_{1}^{i}$ we find that $h_{i} \neq 0$ for all $i$ in some congruence class mod. 4. Now apply Lemma II.6.4.

TheOREM II.6.6. Suppose $P \in A_{k}$ with $1 \in S(P)$. Then $P=Q R$ where $Q=\varphi(z, 1, \cdots, 1)$ for some $z \in k^{*}$, and $1 \notin S(R)$.

Proof. Take $r$ and $\bar{h}$ as in Proposition II.6.5. Choose $z \in k^{*}$ with ord. $z$ $=-r$ and set $Q=\varphi(z, 1, \cdots, 1)$. Suppose that $\bar{u}=\left(\bar{u}_{2}, \cdots, \bar{u}_{g}\right) \in\left(\bar{k}^{*}\right)^{g-1}$ and $\bar{h}(\bar{u}) \neq 0$. We shall show that $\bar{u}$ is either in $\bar{N}_{P Q-1}$ or in $\left(\bar{N}_{P Q}\right)^{-1}$. It will follow from this that either $\bar{N}_{P Q-1}$ or $\bar{N}_{P Q}$ is Zariski-dense. Replacing $z$ by $z^{-1}$ if necessary we can assume $\bar{N}_{P Q^{-1}}$ is dense. By Proposition II.6.1, $1 \notin S\left(P Q^{-1}\right)$. Since $P=Q\left(P Q^{-1}\right)$, the theorem will follow.

To show that $\bar{u}$ is either in $\bar{N}_{P Q^{-1}}$ or in $\left(\bar{N}_{P Q}\right)^{-1}$ lift it to $\left(u_{2}, \cdots, u_{g}\right)$ in $U^{g-1}$ and choose $y$ as in Proposition II.6.5. Set $R=\varphi\left(y, u_{2}, \cdots, u_{\theta}\right)$. Then $\theta_{P}(R)=0$. Now, since ord. $z=-r,\left(y z, u_{2}, \cdots, u_{g}\right)$ is in $U^{g}$ and its image under $\varphi$ is $Q R$. Since $\theta_{P}(R)=0$, Proposition II.5.2, shows that $\theta_{P Q-1}(Q R)=0$ or $\theta_{P Q}\left((Q R)^{-1}\right)=0$. In the first case $\bar{u} \in \bar{N}_{P Q-1}$, in the second case $(\bar{u})^{-1} \in \bar{N}_{P Q}$.

THEOREM II.6.7. In the situation of Theorem II.6.6, $S(Q)=\{1\}$ and $S(R)=S(P)-\{1\}$.

Proof. $Q$ and $R$ have disjoint supports so we may apply Theorem II.4.1.

\section{§II.7. $\varphi$ is surjective}

THEOREM II.7.1. Suppose $k$ is algebraically closed. Then $\varphi: G_{g} / \Gamma$ $\rightarrow A_{k}$ is surjective.

Proof. Suppose $P \in A_{k}$. We show that $P \in \operatorname{Im}(\varphi)$ arguing by induction on the cardinality of $S(P)$. If $S(P)=\varnothing$, Theorem II.3.1 shows that $P \in \varphi\left(U^{g}\right)$. If $S(P) \neq \varnothing$ we may assume $1 \in S(P)$. Since $k$ is algebraically closed we may write $P=Q R$ as in Theorem II.6.6. Theorem II.6.7 and induction conclude the proof.

We next show how to eliminate the hypothesis of algebraic closure.

LEMma II.7.2. Let $0 \neq q \in \mathscr{M}$ and $y \in k^{*}$ with $\mid$ ord. $y \mid \leq \frac{1}{2}$ ord. $q$. Suppose $\alpha \in\{0, \pm 1,2\}, t \in Z$ and $s=t(2 t+\alpha)$ ord. $q+(4 t+\alpha)$ ord. $y$. Then:

(1) if $\alpha=0, s \geq 0$ 
(2) if $\alpha= \pm 1, s \geq-\mid$ ord. $y \mid$. If $\alpha=-1$ and ord. $y>0$, or if $\alpha=1$ and ord. $y<0$ equality occurs only when $t=0$.

(3) if $\alpha=2, s \geq-2 \mid$ ord. $y \mid$. For ord. $y>0$ equality occurs only when $t=-1$. For ord. $y<0$, equality occurs only when $t=0$.

Proof. (1) is clear. To prove (2) and (3) note that $t(2 t+\alpha) \geq 0$. Thus the results hold if ord. $y=0$. If ord. $y>0, s \geq(2 t(2 t+\alpha)+(4 t+\alpha))$ ord. $y$, while if ord. $y<0, s \geq(2 t(2 t+\alpha)-(4 t+\alpha)) \mid$ ord. $y \mid$. The calculation is now straightforward.

LEMmA II.7.3. Suppose $y=\left(y_{1}, \cdots, y_{g}\right) \in G_{g}$ with $\mid$ ord. $y_{j} \mid \leq \frac{1}{2}$ ord. $q_{j}$. Let $S=\left\{j:\right.$ ord. $\left.y_{j} \neq 0\right\}$. Let $\alpha:\{1,2, \cdots, g\} \rightarrow\{0,2\}$ be the map such that $\alpha^{-1}(2)=S$. Then ord. $\theta_{\alpha}(y)=-2 \sum_{j \in S} \mid$ ord. $y_{j} \mid$.

Proof. By (d) of $\S$ II.2, $\theta_{\alpha}(y)=\sum b_{I} y^{I}$ where ord. $\left(b_{I} y^{I}\right)=\sum_{j=1}^{g} s_{j}=\sum_{j=1}^{g}\left(t_{j}\left(2 t_{j}+\alpha_{j}\right)\right.$ ord. $q_{j}+\left(4 t_{j}+\alpha_{j}\right)$ ord. $\left.y_{j}\right)$.

By Lemma II.7.2, $s_{j} \geq-2$ ord. $y_{j} \mid$ for $j \in S$. Thus ord. $\left(b_{I} y^{I}\right)$ $\geq-2 \sum_{j \in S} \mid$ ord. $y_{j} \mid$. Also if equality is to hold we must have $t_{j}=0$ for $j \notin S, t_{j}=-1$ when ord. $y_{j}>0$, and $t_{j}=0$ when ord. $y_{j}<0$. So there is only one monomial for which equality holds, and the lemma follows.

LEMMA II.7.4. Situation as in Lemma II.7.3. Suppose ord. $y_{1} \neq 0$. Define $\beta_{j} \in\{0, \pm 1,2\}$ by setting $\beta_{j}=\alpha_{j}$ if $j>1, \beta_{1}=-1$ if ord. $y_{1}>0$ and $\beta_{1}=1$ if ord. $y_{1}<0$. Then ord. $\theta_{\beta}(y)=\mid$ ord. $y_{1}\left|-2 \sum_{j \in S}\right|$ ord. $y_{j} \mid$.

Proof. Entirely similar to that of Lemma II.7.3.

THEOREM II.7.5. $\varphi: G_{g} / \Gamma \rightarrow A_{k}$ is bijective.

Proof. Theorem II.3.2 shows that $\varphi$ is $1: 1$. To prove ontoness suppose $P \in A_{k}$. Let $L$ be a complete algebraically closed extension of k. By Theorem II.7.1 there is a $y=\left(y_{1}, \cdots, y_{g}\right) \in\left(L^{*}\right)^{g}$ with $\varphi(y)=P$, and we may assume |ord. $y_{j} \mid \leq \frac{1}{2}$ ord. $q_{j}$. Suppose ord. $y_{1} \neq 0$. Define $\alpha$ and $\beta$ as in Lemmas II.7.2 and II.7.3. Then

$$
\text { |ord. } y_{1} \mid=\text { ord. } \theta_{\beta}(y)-\text { ord. } \theta_{\alpha}(y)=\text { ord. }\left(X_{\beta}(P) / X_{\alpha}(P)\right) \text {. }
$$

In particular there exists an $x_{1} \in k^{*}$ such that ord. $x_{1}=$ ord. $y_{1}$. Similarly choose $x_{j} \in k^{*}$ so that ord. $x_{j}=$ ord. $y_{j}$ and let $x=\left(x_{1}, \cdots, x_{g}\right)$. Then 
$y x^{-1} \in U_{L}^{g}$, so $\varphi\left(y x^{-1}\right)$ is a unit point. Since $\varphi\left(y x^{-1}\right)=P \varphi\left(x^{-1}\right)$ it is in $A_{k}$. Thus $P \varphi\left(x^{-1}\right) \in \varphi\left(U_{k}^{g}\right)$ and $P \in \varphi\left(G_{g}\right)$.

III

In this part we show that the map $\varphi: G_{g} / \Gamma \rightarrow A_{k}$ is bijective assuming only that the matrix $\left(\mathscr{A}_{i j}\right)$ is such that each ord. $\mathscr{A}_{i j}$ is rational. We do this by reducing to the diagonal case (cf. § II).

\section{§ III.1. Isogenies}

Let $\left(\mathscr{A}_{i j}\right)$ be a $g \times g$ matrix with entries in $k^{*}$ satisfying the Riemann conditions (i.e. $\left(\mathscr{A}_{i j}\right)$ is symmetric and (ord. $\mathscr{A}_{i j}$ ) is positive definite). Let $S=\left(s_{i j}\right)$ and $T=\left(t_{i j}\right)$ be $g \times g$ matrices over $Z$ such that $S \cdot T=n I$, $n \neq 0$ and let

$$
b_{i j}=\prod_{k, \ell} \mathscr{A}_{k \ell \ell}^{s i k s j}
$$

It is readily seen that the matrix $\left(b_{i j}\right)$ also satisfies the Riemann conditions. Attached to the matrix $\left(\mathscr{A}_{i j}\right)$ are the period vectors $V_{i}$, the group $\Gamma$, the graded ring $R\left(\mathscr{A}_{i j}\right)$ of theta functions, the abelian variety $A$ and the map $\varphi: G_{g} / \Gamma \rightarrow A_{k}$; similarly attached to $\left(b_{i j}\right)$ we have $W_{i}$, $\Gamma^{\prime}, R\left(b_{i j}\right), B$ and $\varphi^{\prime}: G_{g} / \Gamma^{\prime} \rightarrow B_{k}$.

The following identities are obvious:

$$
\begin{gathered}
\prod_{j} b_{i j}^{t_{r j} j}=\prod_{j} \mathscr{A}_{r j}^{n s_{i j}} \\
\prod_{i, j} b_{r_{j}}^{t_{r i} t_{r j}}=\mathscr{A}_{r r}^{n^{2}} .
\end{gathered}
$$

Let $\lambda_{1}, \lambda_{2}: G_{g} \rightarrow G_{g}$ be the maps defined by:

$$
\begin{array}{lll}
\lambda_{1}(x)=\left(y_{1}^{n}, \cdots, y_{g}^{n}\right) & \text { where } & y_{i}=\prod_{j} x_{j}^{s_{i j}} \\
\lambda_{2}(x)=\left(z_{1}, \cdots, z_{g}\right) & \text { where } & z_{i}=\prod_{j} x_{j}^{t_{i j}} .
\end{array}
$$

Proposition III.1.1. $\lambda_{1}$ maps $\Gamma$ into $\Gamma^{\prime}, \lambda_{2}$ maps $\Gamma^{\prime}$ into $\Gamma$ and the composition in either order is the map $x \rightarrow x^{n^{2}}$.

Proof. The image of $V_{r}$ under $\lambda_{1}$ is the vector whose $i$-th component is $\prod_{j} \mathscr{A}_{r j}^{n s s_{j} j}=\prod_{j} b_{i j}^{t_{r j}}$. But this is just the vector $\prod_{j} W_{j}^{t_{r j}}$. Similarly $\lambda_{2}\left(W_{r}\right)=\prod_{j} V_{j}^{n s r j}$. The last assertion is obvious.

For $\theta \in \mathscr{L}$ let $\psi_{1}(X)=\theta\left(Y_{1}^{n}, \cdots, Y_{g}^{n}\right)$ where $Y_{i}=\prod_{j} X_{j}^{s_{i j}}$ and $\psi_{2}(X)$ $=\theta\left(Z_{1}, \cdots, Z_{\theta}\right)$ where $Z_{i}=\prod_{j} X_{j}^{t_{i j}}$. 
Proposition III.1.2. If $\theta \in R_{m}\left(b_{i j}\right)$ then $\psi_{1} \in R_{m n^{2}}\left(\mathscr{A}_{i j}\right)$. If $\theta$ $\in R_{m}\left(\mathscr{A}_{i j}\right)$ then $\psi_{2} \in R_{m n^{2}}\left(b_{i j}\right)$. Consequently $\theta \rightarrow \psi_{1}$ (resp. $\left.\theta \rightarrow \psi_{2}\right)$ gives $a$ graded homomorphism of degree $n^{2} \mu_{1}: R\left(b_{i j}\right) \rightarrow R\left(\mathscr{A}_{i j}\right)$ (resp. $\mu_{2}: R\left(\mathscr{A}_{i j}\right)$ $\left.\rightarrow R\left(b_{i j}\right)\right)$, and the composition (in either order) is the map $\alpha_{n^{2}}: \theta(X)$ $\rightarrow \theta\left(X^{n^{2}}\right)$.

Proof. $\psi_{1}\left(V_{r} X\right)=\theta\left(Z_{1}^{n}, \cdots, Z_{g}^{n}\right)$ where $Z_{i}=\prod_{j}\left(\mathscr{A}_{r j} X_{j}\right)^{s_{i j}}$. It follows from (1) above that $Z_{i}^{n}=\left(\prod_{j} b_{i j}^{t_{r j}}\right) Y_{i}^{n}$ and thus

$$
\left(Z_{1}^{n}, \cdots, Z_{g}^{n}\right)=\left(\prod_{j} W_{\jmath}^{t_{r j}}\right)\left(Y_{1}^{n}, \cdots, Y_{g}^{n}\right)
$$

Since $\theta \in R_{m}\left(b_{i j}\right)$,

$$
\psi_{1}\left(V_{r} X\right)=\left(\prod_{i, j} b_{\imath j}^{t_{r i} t_{r j}}\right)^{-m}\left(\prod_{i} Y_{i}^{-2 m n t_{r i}}\right) \psi_{1}(X)
$$

By (2) this is just $\mathscr{A}_{r r}^{-m n^{2}} X_{r}^{-2 m n^{2}} \psi_{1}(X)$, and so $\psi_{1} \in R_{m n^{2}}\left(\mathscr{A}_{i j}\right)$. Similarly for $\psi_{2}$. The other statements are obvious.

Proposition III.1.3. The homomorphisms of Proposition III.1.2 are finite and induce morphisms of group varieties $\mu_{1}^{*}: A \rightarrow B$ and $\mu_{2}^{*}: B \rightarrow A$.

Proof. Since the composition (in either order) is the map $\alpha_{n^{2}}$ which is finite (cf. Theorem I.1.3), $\mu_{1}$ and $\mu_{2}$ are finite. So we get morphisms of varieties $A \rightarrow B$ and $B \rightarrow A$ which are readily seen to be group variety morphisms.

From Proposition III.1.3 we get homomorphisms $\mu_{1}^{*}: A_{k} \rightarrow B_{k}$ and $\mu_{2}^{*}: B_{k} \rightarrow A_{k}$. The composite map $A_{k} \rightarrow B_{k} \rightarrow A_{k}$ is the map induced by $\alpha_{n^{2}}: R\left(\mathscr{A}_{i j}\right) \rightarrow R\left(\mathscr{A}_{i j}\right)$ which by Theorem I.3.5 is multiplication by $n^{2}$.

\section{$\S$ III.2. $\varphi$ is bijective}

Proposition III.2.1. There is a commutative diagram of maps:

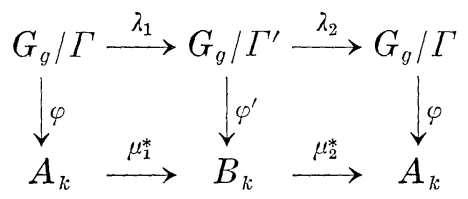

where the $\lambda_{i}$ are induced by the maps of Proposition III.1.1.

Furthermore $\left(\lambda_{2} \circ \lambda_{1}\right)(x)=x^{n^{2}}$ and $\mu_{2}^{*} \circ \mu_{1}^{*}$ is just multiplication by $n^{2}$.

Proof. The commutativity of the diagram follows in a straight- 
forward way from the definition of the maps. The last assertions follow from Propositions III.1.1 and III.1.3.

Now we proceed to show that $\varphi$ is bijective.

Let $\Lambda$ be a subring of the reals, $R$. We say that a $g \times g$ matrix $\mathscr{A}$ over $\boldsymbol{R}$ is $A$-diagonalizable if there exists an invertible matrix $S_{0}$ over $\Lambda$ such that $S_{0} \mathscr{A} S_{0}^{t}$ is diagonal. Let $Z_{\ell}$ denote the localization (not the completion) of $Z$ at the prime $\ell$.

THEOREM III.2.2. Let $\alpha_{i j}=$ ord. $\mathscr{A}_{i j}$ and $\mathscr{A}$ be the matrix $\left(\alpha_{i j}\right)$. Suppose that $\mathscr{A}$ is $\boldsymbol{Z}_{\ell}$-diagonalizable for every prime $\ell$. Then the map $\varphi: G_{g} / \Gamma \rightarrow A_{k}$ is bijective (for the matrix $\left(\mathscr{A}_{i j}\right)$ ).

Proof. Let $S_{0}$ be an invertible matrix over $Z_{\ell}$ diagonalizing $\mathscr{A}$ and let $T_{0}=S_{0}^{-1}$. Replacing $S_{0}$ and $T_{0}$ by integer multiples prime to $\ell$ we get matrices $S$ and $T$ over $Z$ with $S T=n I,(n, \ell)=1$ and $S \mathscr{A} S^{t}$ diagonal. Let $b_{i j}$ be defined as in $\S$ III.1. Then the matrix (ord. $b_{i j}$ ) which is equal to $S \mathscr{A} S^{t}$, is diagonal. So by the main result of $\S$ II, the map $\varphi^{\prime}$ of Proposition III.2.1 is bijective.

Now let $x \in G_{g} / \Gamma$ be such that $\varphi(x)=0$. Then by Proposition III.2.1, $\lambda_{1}(x)=1$ and so $x^{n^{2}}=1$. But $n$ may be taken prime to any $\ell$. Since the $n^{2}$ obtained in this way generate the unit ideal in $Z, x=1$. Similarly, if $P \in A_{k}$ let $P^{\prime}=\mu_{1}^{*}(P)$. Then $P^{\prime} \in \operatorname{Im} . \varphi^{\prime}$ and so $n^{2} P \in \operatorname{Im} . \varphi$. Since $n$ may be choosen prime to any $\ell, P \in \operatorname{Im} . \varphi$ and the theorem is proved.

The following slight modification of Theorem III.2.2 will be useful later.

THEOREM III.2.3. Suppose $\alpha_{i j}=$ ord. $\mathscr{A}_{i j} \in Z$ and generate the unit ideal. Suppose further there exist positive integers $m_{1}, \cdots, m_{s}$ such that $\mathscr{A} \oplus$ diag. $\left(m_{1}, \cdots, m_{s}\right)$ is $Z_{\ell}$-diagonalizable for every prime $\ell$. Then $\varphi$ is bijective (for the matrix $\left(\mathscr{A}_{i j}\right)$ ).

Proof. Since the $\alpha_{i j}$ generate the unit ideal, there exist $q \in k^{*}$ with ord. $q=1$. Then the matrix

$$
\left(\begin{array}{c|ccc}
\mathscr{A}_{i j} & & 1 & \\
\hline 1 & q^{m_{1}} & & 1 \\
& \cdot & \cdot & \\
1 & & & q^{m_{s}}
\end{array}\right)
$$


also satisfies the Riemann conditions and the corresponding order matrix is $\mathscr{A} \oplus$ diag. $\left(m_{1}, \cdots, m_{s}\right)$.

Let $\Gamma_{i}$ be the subgroup of $k^{*}$ generated by $q^{m_{i}}$, and $E_{i}$ the corresponding elliptic curve. Then by Theorem III.2.2 the map

$$
G_{g} / \Gamma \times k^{*} / \Gamma_{1} \times \cdots \times k^{*} / \Gamma_{s} \rightarrow A_{k} \times\left(E_{1}\right)_{k} \times \cdots \times\left(E_{s}\right)_{k}
$$

is bijective. Therefore $\varphi$ is bijective too.

The following simple result will be proved in the appendix.

LEMMA. Let $\left(\alpha_{i j}\right)$ be a symmetric matrix with entries in $Z_{\ell}$. Then:

1) if $\ell \neq 2,\left(\alpha_{i j}\right)$ is $Z_{\ell}$-diagonalizable.

2) if $\ell=2$, there exist integers $m_{1}, \cdots, m_{s}$

which are powers of 2 such that $\left(\alpha_{i j}\right) \oplus$ diag. $\left(m_{1}, \cdots, m_{s}\right)$ is $Z_{\ell}$-diagonalizable.

Let $\left(\mathscr{A}_{i j}\right)$ be our matrix satisfying the Riemann conditions. Combining the above lemma with Theorem III.2.3 we have:

COROLlary 1. If ord. $\mathscr{A}_{i j} \in \boldsymbol{Z}$ and generate the unit ideal, then $\varphi$ is bijective (for the matrix $\left(\mathscr{A}_{i j}\right)$ ).

COROLlary 2. If each ord. $\mathscr{A}_{i j}$ is in $\boldsymbol{Q}$, or less generally, if the value group of the valuation is contained in $\boldsymbol{Q}$, then $\varphi$ is bijective.

\section{Appendix Quadratic forms over $Z_{\ell}$}

Let $R$ be a discrete valuation ring, $M$ a finite free $R$-module and (, ) $: M \times M \rightarrow R$ a symmetric bilinear map. The following lemma is easy linear algebra.

LEMMA 1. Let $n_{1}, \cdots, n_{s} \in M$ and $N$ be the $R$-submodule generated by the $n_{i}$ 's. If det. $\left(\left(n_{i}, n_{j}\right)\right)$ is a unit in $R$, then the $n_{i}$ 's are R-linearly independent and $M=N \oplus N^{\perp}$.

We say that $M$ is decomposable if $M=N \oplus N^{\prime}$ with $N$ and $N^{\prime}$ nonzero submodules of $M$ and orthogonal; $M$ is diagonalizable if it is the orthogonal sum of 1-dimensional submodules; and $M$ is primitive if there exist $m, m^{\prime} \in M$ with $\left(m, m^{\prime}\right)$ a unit in $R$.

THEOREM 1. If 2 is a unit in $R$, then $M$ is diagonalizable.

Proof. We may assume $M$ primitive. Let $m, m^{\prime} \in M$ with $\left(m, m^{\prime}\right)$ a 
unit. Then $\left(m+m^{\prime}, m+m^{\prime}\right)=(m, m)+\left(m^{\prime}, m^{\prime}\right)+$ unit. So there exists $n \in M$ with $(n, n)$ a unit. By Lemma $1, M=R n \oplus(R n)^{\perp}$ and we use induction on the dimension.

CoRollaRy 1. Let $\mathscr{A}$ be a symmetric matrix over $Z_{\ell}(\ell \neq 2)$. Then there exists an invertible matrix $S$ over $Z_{\ell}$ such that $S \mathscr{A} S^{t}$ is diagonal.

Suppose now that 2 is not a unit in $R$.

Lemma 2. If $M$ is primitive and indecomposable, then $\operatorname{dim} . M \leq 2$.

Proof. If there exists $m \in M$ with $(m, m)$ a unit then by Lemma 1 , $M=R m \oplus(R m)^{\perp}$. So $M=R m$ and $\operatorname{dim} . M=1$. Suppose that $(m, m)$ is in the maximal ideal of $R$ for all $m$. Choose $m_{1}$ and $m_{2}$ with $\left(m_{1}, m_{2}\right)$ a unit. By Lemma 1 and indecomposibility, $M=R m_{1}+R m_{2}$.

THEOREM 2. For any $M$ there exists a diagonalizable $R$-module $N$ such that the orthogonal direct sum of $M$ and $N$ is diagonalizable.

Proof. We may assume $M$ primitive and indecomposable. By Lemma 2 we may assume $M$ generated by $e_{1}$ and $e_{2}$ with $\left(e_{1}, e_{1}\right),\left(e_{2}, e_{2}\right)$ in the maximal ideal and $\left(e_{1}, e_{2}\right)$ a unit. Replacing $e_{2}$ by a multiple we may assume $\left(e_{1}, e_{2}\right)=-1$. Let $N=R e_{3}$ with $\left(e_{3}, e_{3}\right)=1$. Then $\left(e_{1}+e_{3}, e_{2}+e_{3}\right)$ $=0$. Since $\left(e_{1}+e_{3}, e_{1}+e_{3}\right)$ and $\left(e_{2}+e_{3}, e_{2}+e_{3}\right)$ are units we conclude from Lemma 1 that $M \oplus N$ admits an orthogonal basis consisting of $e_{1}+e_{3}, e_{2}+e_{3}$ and one other vector.

Remark. The proof of Theorem 2 shows the following: if $\pi$ is a generator of the maximal ideal of $R$, then $N$ can be choosen to have the form $\oplus R u_{i}$ with $\left(u_{i}, u_{j}\right)=\pi^{n_{i}} \delta_{i j}$.

Taking $R=Z_{2}$ and $\pi=2$ we have:

COROLlaRY 2. Let $\mathscr{A}$ be a symmetric matrix over $Z_{2}$ Then there exist $m_{1}, \cdots, m_{s}$ which are powers of 2 such that the matrix $\mathscr{A} \oplus \operatorname{diag} .\left(m_{1}\right.$, $\cdots, m_{s}$ ) is $Z_{2}$-diagonalizable.

\section{REFERENCES}

[1] Gerritzen, L. On non-archimedean representation of abelian varieties. Math. Ann., vol. 196 (1972), pp. 323-346.

[2] McCabe, J. P-adic theta functions. Ph.D. thesis, Harvard University (1968), unpublished.

[ 3 ] Morikawa, H. On theta functions and abelian varieties over valuation fields of rank one. I, II Nagoya Math. Jour. vol. 20 (1962), pp. 1-27 and pp. 231-250. 
[4] Mumford, D. An analytic construction of degenerating abelian varieties over complete local rings. Composito Math. vol. 24 (1972), pp. 129-174 and pp. 239-272.

[ 5 ] Raynaud, M. Variétés abéliennes et géométrie rigide. Actes Congress Intern. Math. 1 (1970), pp. 473-477.

[6] Roquette, P. Analytic theory of elliptic functions over local fields. Vandenhoeck and Ruprecht in Göttingen (1970).

Departamento de Matemáticas Centro de Investigación, I.P.N.

México, D.F. México 